APR 211905

\author{
MII \\ University of California \\ Ernest 0 . Lawrence \\ Radiation Laboratory
}

UCRL-12207

\title{
DISPERSION RELATION FOR RELATIVISTIC STREAMS OF FINITE RADIUS
}

\author{
PATENT CIEARANCE OBTANED. PEEEGLISETS TS

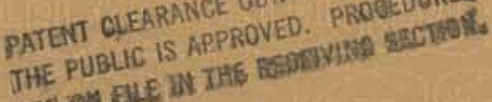 \\ BEFE
}




\section{DISCLAIMER}

This report was prepared as an account of work sponsored by an agency of the United States Government. Neither the United States Government nor any agency Thereof, nor any of their employees, makes any warranty, express or implied, or assumes any legal liability or responsibility for the accuracy, completeness, or usefulness of any information, apparatus, product, or process disclosed, or represents that its use would not infringe privately owned rights. Reference herein to any specific commercial product, process, or service by trade name, trademark, manufacturer, or otherwise does not necessarily constitute or imply its endorsement, recommendation, or favoring by the United States Government or any agency thereof. The views and opinions of authors expressed herein do not necessarily state or reflect those of the United States Government or any agency thereof. 


\section{DISCLAIMER}

Portions of this document may be illegible in electronic image products. Images are produced from the best available original document. 


\section{UNIVERSITY OF CALIFORNIA}

Lawrence Radiation Laboratory

Livermore, California

"

AEC Contract Nọ. W-7405-eng - 48

DISPERSION RELATION FOR RELATIVISTIC STREAMS OF FINITE RADIUS

Ronald W. Landau

January 4, 1.965

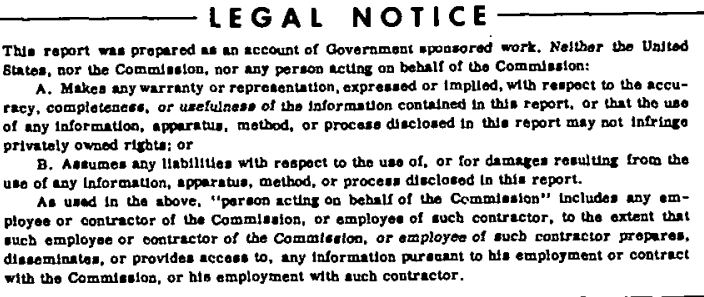

with the Commieston, or his employment with euch contreter. 


\section{INTRODUCTION}

Many authors have considered the problem of longitudinal plasma oscillations. $^{1-6}$ These models have included plasmas both hot and cold, streaming and stationary, relativistic and nonrelativistic, and in cylindrical and infinite geometries. Nevertheless, as pointed out by Stix, ${ }^{7}$ instabilities occur where theory predicts stability. The problem of radiation from these plasma oscillations has also been considered. ${ }^{3,8,9}$ The crucial question of the excitation of the radiation remains open.

We have therefore re-examined the problem in more detail and examined the two-stream longitudinal instability for a finite system. A general dispersion relation has in fact been obtained by us (Eqs. (159)-(161)). In this paper we content ourselves with the presentation of the derivation and its relationship with previous work. The computational details of the solutions will be left for the future.

A considerable unification of previous results has thereby been accomplished. It is hoped that solutions of the equation will show that the two-stream instability can drive the plasma radiation.

The model considered is that of a cylinder of radius a and infinite length populated with charged particles and immersed in an infinitely strong magnetic field, directed along the cylinder axis, so that only longitudinal motion occurs. The magnetic field thus acts only as a constraint. Other discussions of longitudinal oscillations dispense with this artifice and consider only motion parallel to some vector $\mathfrak{k}_{\mathrm{z}}$, which in our case is along the cylinder axis.

Each charged particle stream is assumed to be uniform in density across the cylinder and may be moving relativistically. The velocity distribution of 
each stream is also uniform throughout the cylinder, but is assumed sufficiently narrow so that the beam may be characterized by one value of $\gamma$ (the relativistic factor). It is otherwise arbitrary. Each beam is characterized by a particle density, particle mass charge sign, velocity distribution, and average velocity. These five quantities may differ for each beam, but the radius of each beam is the same. The cylinder is isolated in space (see Fig.1).

This system is in neutral equilibrium. At time $t=0$ the system is disturbed. The subsequent time evolution of the fields and other variables is obtained by the Laplace transform and the assumption that all variables are first order small. The result may be expressed in the following form. The system behaves in time like $\sum a_{n} e^{-i \omega_{n} t}$, where the discrete $\omega_{n}$ are solutions of a dispersion relation. There is also a term which decays with time, and for given $k$ becomes negligible as to $t>1 / k c \equiv 1 / \omega_{k}$. This term apparently establishes causality, i. e., that no signal can travel faster than $c$, so that at time $t=\tau$, for example, there is no disturbance farther than $c \tau$ away from the edge of the plasma cylinder. This term arises from a branch cut integral and is apparently related to the branch cut integrals discussed by Brillouin 10 and Stratton ${ }^{11}$ in their analysis of signal velocities in dispersive media.

The dispersion relation obtained may be simplified in two limits. One where $\kappa a \gg 1, \kappa a \equiv\left[1-(\omega / k c)^{2}\right]^{1 / 2} \mathrm{ka}$, and the other where $k a \ll 1$. Thus the form of the equations depends not only on $\mathrm{ka}$, but also on the phase velocity of the wave. The "thin beam" equation $(k \mathrm{a}<<1)$ will then be obtained even for $\mathrm{ka} \gg 1$, if $\omega / \mathrm{kc} \approx 1$. All quantities behave like $\mathrm{e}^{i k z}$ which defines $\mathrm{k}$. 
The physical significance of $k$ when $\omega / k c<1$ is that $k$ is the wave vector measured in a system moving with the wave velocity $\omega / \mathrm{kc}$. Only in this system can the longitudinal electric field be obtained from Poisson's equation, as the charge distribution is stationary. (The particles move, but the charge distribution in this moving frame is stationary.) Since the longitudinal electric field is Lorentz invariant, this field is the same in the lab frame and hence is the physically significant quantity.

These ideas immediately suggest the existence of two branches of the dispersion relation. One where (Real part $\omega) / k c<1$ and the other where $\operatorname{Re}(\omega) / \mathrm{kc}>1$. For the former case, we have the situation just discussed, where a Lorentz transformation may be made to a system where the charges are independent of time though varying in $z$. For the latter case a Lorentz transformation may be made to a system where the currents are independent of $z$, though varying in time. These ideas are covered in more detail by Landau and Schmidt, 12

For the case where the phase velocity of the wave is larger than $c$, in the lab frame, the waves will radiate by a Cerenkov-type process. ${ }^{9}$ The twostream instability mechanism may provide an energy source for this radiation. Unfortunately, our dispersion relation has not, as yet; been solved to see if the above qualitative arguments are true.

From our dispersion relation the works of other authors may be obtained. The results of Jackson, ${ }^{2}$ Bludman et al. , 4 and others for the infinite beam case may be recovered when $\kappa a \gg 1$. As mentioned, these results are now seen to be invalid when $\omega / k c \approx 1$. It is moreover clear that a discontinuity in the equations exists when $\omega / \mathrm{kc} \approx 1$ and that only solutions with complex $\omega$ are found if $\omega / \mathrm{kc}>1$. This is not found in the strictly one-dimensional case considered by Buneman, 13 where the waves are undamped. 
The thin beam results of Sturrock ${ }^{6}$ and Finkelstein and Sturrock ${ }^{14}$ are also recovered when $\kappa a<1$. It is seen that the results obtained by these authors are valid only if $\omega / \mathrm{kc} \ll 1$.

When the temperature of the plasma is set equal to zero, the results of Budker ${ }^{5}$ are obtained. His treatment is more restrictive than ours in that. only the $\mathrm{m}=0$ angular independent perturbations are considered. The derivation of Budker's results has heretofore been unavailable in the literature.

\section{THE EQUATIONS}

Although our final treatment will discuss $\mathrm{N}$ streams, for the moment only one stream will be assumed. Collisions will be neglected so that the system may be described by the collisionless Boltzmann equation, or kinetic equation. The variables are the canonical momenta and the coordinates $r, \theta$, and $\mathrm{z}$ for a cylindrical system. The assumption of very large axial magnetic field allows a considerable simplification of the equations because then only longitudinal particle motion is permitted. If we further restrict our consideration to small perturbations from an equilibrium state, the equations may be linearized and explicit expressions obtained for the desired quantities. The desired equations will be derived in the following sections.

\subsection{The Kinetic Equation}

The kinetic equation for the distribution function $f$ is given by

$$
\frac{\partial \mathrm{f}}{\partial \mathrm{t}}+\dot{\mathrm{r}} \frac{\partial \mathrm{f}}{\partial \mathrm{r}}+\dot{\theta} \frac{\partial \mathrm{f}}{\partial \theta}+\dot{z} \frac{\partial \mathrm{f}}{\partial \mathrm{z}}+\dot{\mathrm{p}}_{\mathrm{r}} \frac{\partial \mathrm{f}}{\partial \mathrm{p}_{\mathrm{r}}}+\dot{\mathrm{p}}_{\theta} \frac{\partial \mathrm{f}}{\partial \mathrm{p}_{\theta}}+\dot{\mathrm{p}}_{\mathrm{z}} \frac{\partial \mathrm{f}}{\partial \mathrm{p}_{\mathrm{z}}}=0
$$


where $\mathrm{f}$ is defined by

$$
\mathrm{n}=\int \mathrm{f} d \mathrm{p}_{\mathrm{r}} \frac{\mathrm{dp} \mathrm{p}_{\theta}}{\mathrm{r}} \mathrm{dp_{ \textrm {z } }}
$$

and $\mathrm{n}$ is the particle density. This equation is relativistically correct ${ }^{15}$ if the coefficients are obtained from a relativistic Hamiltonian. The coefficients are

$$
\dot{q}_{i}=\frac{\partial H}{\partial p_{i}}, \quad \dot{p}_{i}=-\frac{\partial H}{\partial p_{i}}, p_{i}=p_{r}, p_{\theta}, p_{z} ; q_{i}=r, \theta, z,
$$

where $\mathrm{H}$ is given by

$H=c\left[\left(p_{r}-\frac{e}{c} A_{r}\right)^{2}+\left(\frac{p_{\theta}}{r}-\frac{e}{c} A_{\theta}\right)^{2}+\left(p_{z}-\frac{e}{c} A_{z}\right)^{2}+\left(m_{0} c\right)^{2}\right]^{1 / 2}+e \phi$.

The potentials $\phi$ and $A$ are due to the external fields, which are known, and the self-fields of the beam particles. For the self-fields we shall use the retarded potentials. We then have

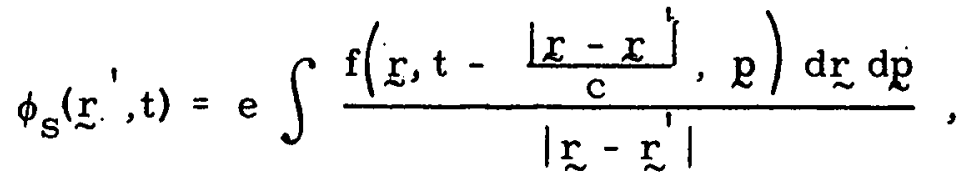

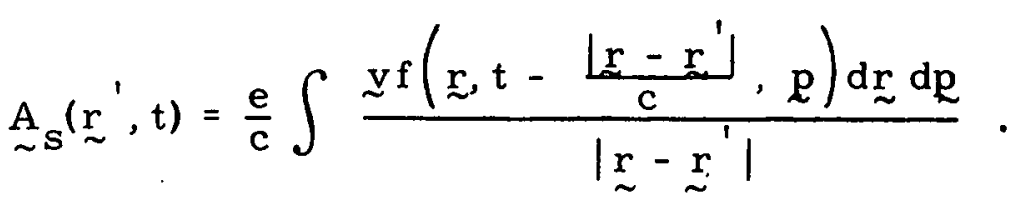

The abbreviation $d \underset{\sim}{r} d \underset{z}{ }=d r d \theta d z d p_{r} d p_{\theta} d p_{z}$ is used. The velocity $v=\{\dot{r}, r \dot{\theta}, \dot{z}\}$ is also evaluated at the retarded time.

The coefficients of Eq. (1), the kinetic equation, have now been given, so that Eq. (1) is a complicated integro-differential equation for $f$. From this equation we see that the variables of our problem are $r, \theta, z, t, p_{r}, p_{\theta}$, $\mathrm{p}_{z}$. All other quantities, e.g., $\dot{\mathrm{r}}, \dot{\theta}, \dot{\mathrm{p}}_{\mathrm{z}}$, are to be thought of as functions 
of these variables. Moreover, the above system is exact, save for the neglect of collisions, and obviously includes Maxwell's equations.

In the next section we show how the assumption of large axial magnetic field simplifies the above system.

\subsection{The One-Dimensional Kinetic Equation}

The explicit equations for $\dot{r}$ and $\dot{\theta}$ are now needed. By Eqs. (3) and (4),

$$
\dot{r}=\frac{1}{m}\left(\frac{p_{r}}{m}-\frac{e}{c} A_{r}\right), \dot{\theta}=\frac{1}{m}\left(\frac{p_{\theta}}{r}-\frac{e}{c} A_{\theta}\right),
$$

where $\mathrm{mc}^{2}=\mathrm{H}$. From orbit theory we know that a longitudinal magnetic field impedes transverse particle motion. If the external longitudinal field is infinite, $\dot{r}$ and $\dot{\theta}$ equal zero. If the function $\mathrm{f}$ is reasonable smooth, then $\partial f / \partial r$ and $\partial f / \partial \theta$ are finite within the beam (except for the singularity of $\partial f / \partial r$ at $r=a$, a point ' of zero measure which will not concern us). Hence the terms $\dot{\mathrm{r}} \partial \mathrm{f} / \partial \mathrm{r}$ and $\dot{\theta} \partial \mathrm{f} / \partial \theta$ in the kinetic equation equal zero.

We may further simplify the kinetic equation by integrating it over $\mathrm{dp}_{\theta} \mathrm{dp} \mathrm{p}_{\mathrm{r}}$. Since $\mathrm{A}_{\mathrm{r}}$ and $\mathrm{A}_{\theta}$ are functions of position only, and $\dot{\mathrm{r}}$ and $\dot{\theta}$ equal zero, by Eq. (7) $p_{\theta}$ and $p_{r}$ become functions of $r, \theta$, and $z$. With this substitution, the functions $\dot{\mathrm{p}}_{\mathrm{r}}$ and $\dot{\mathrm{p}}_{\theta}$, obtained from Eq. (3), are no longer functions of $\mathrm{p}_{\mathrm{r}}$ and $\mathrm{p}_{\theta}$ and may be taken out of the integral sign so that

$$
\int \dot{\mathrm{p}}_{\theta} \frac{\partial \mathrm{f}}{\partial \mathrm{p}_{\theta}}=\dot{\mathrm{p}}_{\theta} \int \frac{\partial \mathrm{f}}{\partial \mathrm{p}_{\theta}} \mathrm{dp} \mathrm{p}_{\theta}=\dot{\mathrm{p}}_{\theta}[\mathrm{f}]_{-\infty}^{\infty}=0
$$

because $f$ vanishes at the limits. The term involving $\dot{p}_{r}$ is similarly zero. Adopting the definition 
$-8-$

$$
\bar{f}=\int f d p_{r} \frac{d p_{\theta}}{r}
$$

allow F as. (1), (B), and (6) to be written in terms of 7 . (Wo shall however drop the bar in the following.) Thus Eq. (1) becomes

$$
\frac{\partial f}{\partial t}+\dot{z} \frac{\partial f}{\partial z}+\dot{p}_{z} \frac{\partial f}{\partial p_{z}}=0
$$

where $f$ is defined by

$$
n=\int f d p_{z}
$$

instead of Eq. (2), and $\mathrm{n}$ is still the particle density. The Hamiltonian may now be written, since $\dot{r}, \dot{\theta}=0$, and using Eq. (7), as

$$
H=c\left[\left(p_{z}-\frac{e}{c} A_{z}\right)^{2}+\left(m_{0} c\right)^{2}\right]^{1 / 2}+e \phi \text {. }
$$

The coefficients $\dot{z}$ and $\dot{p}_{z}$ may be obtained through Eq. (3) and the new Hamiltonian. They are

$$
\dot{z}=\frac{1}{m}\left(p_{z}-\frac{e}{c} A_{z}\right)
$$

and

$$
\dot{\mathrm{p}}_{\mathrm{z}}=\mathrm{e} \frac{\dot{\mathrm{z}}}{\mathrm{c}} \frac{\partial \mathrm{A} z}{\partial \mathrm{z}}-\mathrm{e} \frac{\partial \phi}{\partial \mathrm{z}}
$$

where

$$
m c^{2}=H-e \phi=c\left[\left(p_{z}-\frac{e}{c} A_{z}\right)^{2}+\left(m_{0} c\right)^{2}\right]^{1 / 2}
$$

The equations for the self-fields, Ens. (5) and (6), become

$$
\phi\left(\underline{\sim}^{\prime}, t\right)=e \int \frac{f\left(r, t-\frac{\left|\underline{r}-\underline{r}^{\prime}\right|}{c}, p_{z}\right)}{\left|\underline{r}-\underline{\sim}^{\prime}\right|} r d r d \theta d z d p_{z^{\prime}}
$$




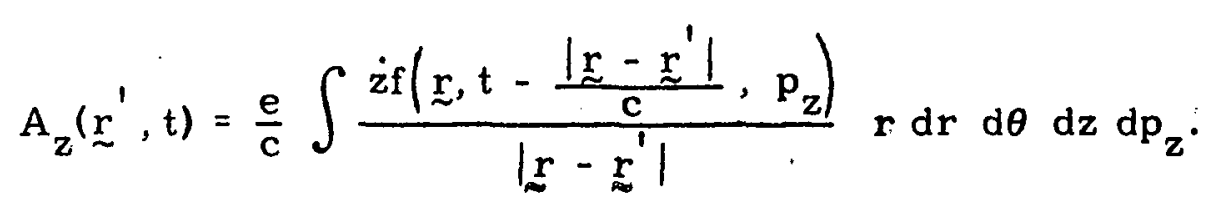

The subscripts $s$ of Eqs. (5) and (6) have been dropped because the fields $A_{z}$ and $\phi$ are all self-fields. Although there is an external $B_{z}$ magnetic field, in the Lorentz gauge, it does not contribute to $A_{z}$. There are no other external magnetic or electric fields.

This completes the specification of the one-dimensional kinetic equation. In the following sections these equations will be further simplified by assuming that the perturbations in the equilibrium distribution are small and that the temperature spread of the relativistic beam is small..

\subsection{The Linearized Kinetic Equation}

The first approximation we shall make is to assume that the changes in our equilibrium configuration are small. The distribution function may then be written as

$$
f=f_{0}+f_{1}
$$

where $f_{0}$ describes the equilibrium configuration, and $f_{1}<<f_{0}$, in some operational sense, describes small departures from equilibrium. Note that $f_{0}=f_{0}\left(r, p_{z}\right)$ and $f_{1}=f_{1}\left(r, \theta, z, t, p_{z}\right)$. Writing then Eq. (10), using Eq. (18) for $f$, keeping only terms proportional to $f_{1}$, and dropping terms proportional to $\left(f_{1}\right)^{2}$ and higher powers, we obtain

$$
\frac{\partial f_{1}}{\partial t}+\dot{z}_{0} \frac{\partial f_{1}}{\partial z}+\dot{p}_{z}^{1} \frac{\partial f_{0}}{\partial p_{z}}=0
$$


Here $\dot{z}_{0}$ denotes the function $\dot{z}$, where only terms linear in $f_{0}$ are kept,

while $\dot{\mathrm{p}}_{\mathrm{z}}{ }^{1}$ denotes the function $\dot{\mathrm{p}}_{\mathrm{z}}$, where only terms linear in $\mathrm{f}_{1}$ are kept. (There are no terms linear in $f_{0}$ contained in $\dot{p}_{z}$ ) The derivation of Eq. (19) is evident. One need only note that

$$
\frac{\partial \mathrm{f}_{0}}{\partial \mathrm{t}} \text { and } \frac{\partial \mathrm{f}_{0}}{\partial \mathrm{z}}=0 \text {. }
$$

In the next two sections we shall evaluate the quantities $\dot{z}_{0}$ and $\dot{\mathrm{p}}_{\mathrm{z}}{ }^{1}$ explicitly.

\subsection{The Coefficient $\dot{\mathrm{z}}_{0}$}

The exact expression for $\dot{z}$ is given by Eqs. (13) and (15). We will expand this quantity using the two assumptions that the velocity spread is small and that the perturbation in the self-field is small. Although, to obtain $\dot{z}$ in Eq. (19), it is sufficient to obtain $\dot{z}$ to zero order, we will need the first order terms in order to obtain $\dot{\mathrm{p}}_{\mathrm{z}}{ }^{1}$ correctly to first order.

Before performing the expansion, let us examine the terms in Eq. (13). The variation of $\mathrm{p}_{z}$ in Eq. (13) gives a velocity spread at any one spatial point. The term $A_{z}$, the self-field, has contributions from the zero order distribution, which we shall denote $A_{0}$, and from the perturbed distribution, to be labeled $A_{1}$.

We will expand $\dot{z}$ about the quantity $\overline{\dot{z}}$, defined by

$$
\overline{\dot{z}}=\frac{1}{\bar{m}}\left(\bar{p}_{z}-A_{0}\right) \text {. }
$$

The quantity $\bar{p}_{z}$ is defined as the average value of $p_{z}$, i.e., 


$$
\bar{p}_{z}=\frac{1}{n_{0}} \int p_{z} f_{0} d p_{z}
$$

whlle $\bar{m}$ is defined as

$$
\overline{\mathrm{m}}=\frac{1}{\mathrm{c}}\left[\left(\overline{\mathrm{p}}_{\mathrm{z}}-\frac{\mathrm{e}}{\mathrm{c}} \cdot \mathrm{A}_{0}\right)^{2}+\left(\mathrm{m}_{0} \mathrm{c}\right)^{2}\right]^{1 / 2} \text {. }
$$

The dispersion relation may be obtained in a simple form when $\bar{z}$ is independent of radius. Since, in general $A_{0}$ is a function of radius, we will assume that the radial dependence of $\bar{p}_{z}$ cancels that of $A_{0}$, so that $\bar{z}$ is a constant. This is the reason for expanding $\dot{z}$ about $\bar{z}$ as defined above.

The expansion of $\dot{z}$ now proceeds trivially, using Eqs. (13) and (15). To first order in $P=p_{z}-\bar{p}_{z}-\frac{e}{c} A_{1}$ we obtain

$$
z=\bar{z}+\left(\frac{\partial \dot{z}}{\partial p_{z}}\right)_{P=0}\left[p_{z}-\bar{p}_{z}-\frac{e}{c} A_{1}\right] \text {. }
$$

By Eqs. (13) and (15),

$$
\begin{aligned}
\frac{\partial \dot{z}}{\partial p_{z}} & =\frac{\partial}{\partial p_{z}}\left(\frac{1}{m}\right) \cdot\left(\dot{p}_{z}-\frac{e}{c} A_{z}\right)+\frac{1}{m} \frac{\partial}{\partial p_{z}}\left(p_{z}-\frac{e}{c} A_{z}\right) \\
& =-\frac{1}{m^{3} c^{2}}\left(p_{z}-\frac{e}{c} A_{z}\right)^{2}+\frac{1}{m}
\end{aligned}
$$

so that

$$
\left(\frac{\partial \dot{z}}{\partial p_{z}}\right)_{P=0}=-\frac{(\bar{z})^{2}}{\bar{m} c^{2}}+\frac{1}{\bar{m}}=\frac{1}{\bar{m}}\left[1-\frac{(\bar{z})^{2}}{c^{2}}\right]=\frac{1}{(\bar{\gamma})^{3} m_{0}}
$$

where

$$
(\bar{\gamma})^{2}=\frac{1}{1-\frac{(\overline{\dot{z}})^{2}}{c^{2}}}
$$


Since this expansion is to first order in $f_{1}$, we assumed above that $A_{z}=A_{0}$ $+A_{1}$, and we neglected higher order terms. The desired expression for $i$ is therefore

$$
\dot{z}=\bar{z}+\frac{1}{(\bar{\gamma})^{3} m_{0}}\left(p_{z}-\bar{p}_{z}-\frac{e}{c} A_{1}\right) .
$$

This expression is valid when $p_{z}-\bar{p}_{z}$ is small. Consideration of higher order terms in the expansion shows that they are negligible if $\Delta \gamma / \gamma \ll 1$. Here $\gamma^{2}=\left[1-(\dot{z} / \mathrm{c})^{2}\right]^{-1}$ and is a function of $\mathrm{p}_{\mathrm{z}}-\overline{\mathrm{p}}_{\mathrm{z}}$ through Eq. (26). For this case all particles in the beam may be characterized by one value of $\gamma$, so that the bar on $\bar{\gamma}$ in Eq. (26) may be dropped.

We shall show now that we may make a change of variables in Eq. (19) and in the explicit definitions of the coefficients. This simplifies calculations if we assume that $f_{0}$ may be written in the form $f_{0}=f_{0}\left(p_{z}-\bar{p}_{z}\right)$. By Eq. (26), this ensures that the initial distribution has a uniform velocity throughout the cylinder (since $\bar{z}$ is independent of $r$ ). The variable $p_{z}$ enters Eq. (19) also through $\dot{z}$, defined in Eq. (26), again in the combination $p_{z}-\bar{p}_{z}$. In fact $p_{z}$ enters Eq. (26) only in this combination. We may therefore make the change of variable

$$
\mathrm{p}=\mathrm{p}_{\mathrm{z}}-\overline{\mathrm{p}}_{\mathrm{z}}
$$

Noting that $d p=d p_{z}$, the linearized kinetic equation becomes

$$
\frac{\partial f_{1}}{\partial t}+\dot{z}_{0} \frac{\partial f_{1}}{\partial z}+\dot{p}_{z} \frac{\partial f_{0}}{\partial p}=0 .
$$

By Eq. (26) we now have

$$
\dot{\mathrm{z}}_{0}=\overline{\mathrm{z}}+\frac{1}{\gamma^{3} \mathrm{~m}_{0}} \mathrm{p} \equiv \mathrm{v} .
$$


We note that $\dot{p}_{z}$ is a function of a number of variables including $p_{z}$ and hence is still called $\dot{\mathrm{p}}_{\mathrm{z}}$.

It is instructive to compare our equations with the usuaf dert vations, $1,2,4$ The difference is in our use of $\dot{p}_{z}$ in place of eE (where $E$ is the electric field), and $p$ in place of the velocity. The quantity $\dot{p}_{z}$ in Eq. (28) is the canonical momentum and is related to the more commonly used linear momentum, miz, through Eq. (13). Hence $\dot{\mathrm{p}}_{\mathrm{z}}=\frac{\mathrm{d}}{\mathrm{dt}}(\mathrm{m} \dot{\mathrm{z}})+\frac{\mathrm{e}}{\mathrm{c}} \ddot{\mathrm{A}}_{\mathrm{z}}$. Ordinarily one writes $\frac{d}{d t}(m i)=e E$. Here, however, $\dot{p}_{z}=e E+\frac{e}{c} \dot{A}_{z}$ which shows why our equations differ.

Note, too, the relationship of $\mathrm{p}$ in Eq. (27) to the linear momentum. Through Eqs. (27), (20), and (13), we may write

$$
\mathrm{p}=\mathrm{p}_{\mathrm{z}}-\overline{\mathrm{p}}_{\mathrm{z}}=\mathrm{m} \dot{\mathrm{z}}+\frac{\mathrm{e}}{\mathrm{c}} \mathrm{A}_{\mathrm{z}}-\overline{\mathrm{p}}_{\mathrm{z}}=\mathrm{m} \dot{\mathrm{z}}+\frac{\mathrm{e}}{\mathrm{c}} \mathrm{A}_{0}-\overline{\mathrm{p}}_{\mathrm{z}}=\mathrm{m} \dot{\mathrm{z}}-\overline{\mathrm{m}} \dot{\mathrm{z}},
$$

and hence, at least to lowest order, $p$ differs from the linear.momentum by a constant.

\subsection{The Coefficient $\dot{\mathrm{p}}_{\mathrm{z}}{ }^{1}$}

Having now evaluated $\dot{z}_{0}$ explicitly, we next turn to the second coefficient in Eq. (28), $\dot{\mathrm{p}}_{z}{ }^{1}$. Since the zero-order self-field $A_{0}$ is independent of $z\left(\phi_{0}=0\right)$, by Eq. (14), $\dot{p}_{z}$ is a first order quantity. Hence we may rewrite Eq. (14) as

$$
\dot{\mathrm{p}}_{\mathrm{z}}^{1}=\mathrm{e} \frac{\dot{z}_{0}}{\mathrm{c}} \frac{\partial \mathrm{A}_{1}}{\partial z}-\mathrm{e} \frac{\partial \phi_{1}}{\partial z} .
$$

The coefficient $\dot{z}$ in Eq. (14) is evidently of zero order, and has therefore been written as $\dot{z}_{0}$ in Eq. (30). It is given by Eq. (29). The second term, $\phi_{1}$, given by Eq. (16), is 


$$
\phi_{1}\left(r^{\prime}, \theta^{\prime}, z^{\prime}, t\right)=e \int \frac{f_{1}\left(r, \theta, z, t-\frac{\left|r-r^{\prime}\right|}{c}, p\right)}{\mid r-\underset{\sim}{r^{\prime} \mid}} r d r d \theta d z d p
$$

To obtain the corresponding expression for $A_{1}$ requires more care because of the product term. $z$ if in the integrand of Eq. (17). By Eqs. (18), (26), and (27), we have for this term

$$
\begin{aligned}
\dot{z} f_{f} & =\left[\left(\dot{z}+\frac{p}{\gamma^{3} m_{0}}\right)-\frac{e \dot{A}_{1}}{c \gamma^{3} m_{0}}\right]\left(f_{0}+f_{1}\right) \\
& =\left(v-\frac{e A_{1}}{c \gamma^{3} m_{0}}\right) \cdot\left(f_{0}+f_{1}\right)
\end{aligned}
$$

using also Eq. (29). Carrying out the multiplication we obtain four terms; one $\sim f_{0}$, two $\sim f_{1}$, and one $\sim\left(f_{1}\right)^{2}$. The term $\sim_{f_{0}}$ gives $A_{0}$. The desired term is $A_{1} \sim f_{1}$ 'so we drop the higher order term $\sim\left(f_{1}\right)^{2}$. Combining the desired terms $\sim_{1}$ in the integrand of Eq. (17) gives finally for $A_{1}$,

$$
A_{1}\left(\sim_{\sim}^{\prime}, t\right)=\frac{e}{c} \int \frac{\left(\dot{v f}_{1}-\frac{e A_{1} f_{0}}{c \gamma^{3} m_{0}}\right)}{\left|\underset{\sim}{r}-{\underset{\sim}{r}}^{\prime}\right|} r d r d \theta d z d p .
$$

The desired equations have now been obtained. These equations are valid for a single beam, but may easily be generalized to many beams. This will be done later.

The system that we shall solve includes Eqs. (28), (29), (30), (31), and (33). The function $f_{0}(p)$ is independent of $r$ for $r \leq a$, i. e., within the beam, and equals zero for $r>$ a. Also, by Eq. (21),

$$
\int p f_{0}(p) d p=0 \text {. }
$$


In spite of its apparent complexity, this system of equations may be solved exactly by the methods of Fourier, Fourier-Besse1, and Laplace transforms. The following sections will discuss this method of solution.

\section{THE SOLUTION}

In the following sections the solution of the above system of equations is elaborated. The general procedure may be sketched. The unknown functions, $\phi_{1}, A_{1}, f_{1}$, and $\dot{p}_{z}{ }^{1}$ are written as transforms in the variables $r, \theta, z$, and $t$ : These functions are inserted into the right-hand side of Eqs. (31) and (33), and the integrations are performed explicitly. If the transforms are written in a suitable manner, it is possible to solve Eqs. (31) and (33) and to obtain the transforms $\phi^{\top}$ and $A^{\tau}$ of $\phi_{1}$ and $A_{1}$ in terms of $f^{\top}$, the transform of the perturbed distribution. By taking the transform of the kinetic equation, Eq. (28), one may obtain $\mathrm{f}^{\tau}$ in terms of $\dot{\mathrm{p}}_{\mathrm{z}}{ }^{\boldsymbol{T}}$ and the initial conditions. On taking the transform and substituting the previous results, one obtains an integral equation for $\dot{p}_{z}^{\tau}$. In the case of $N$ streams the above procedure leads to $N$ integral equations. The solution of this set of equations is easily obtained; it requires solving $2 \mathrm{~N}$ linear algebraic equations in $2 \mathrm{~N}$ unknowns by the methods of ordinary algebra. The transforms of all the quantities are thereby obtained in terms of the initial conditions and the problem is solved, at least in principle. The extraction of a useful dispersion relation will be deferred to Chapter 4 . 


\subsection{The Fourier-Bessel-Laplace Transform}

We will show here the desired expansion formulae for the unknown functions. The time dependence will be expanded uaing the Laplace transform, the $\mathrm{z}$-dependence using the Fourier transform, and the $\theta$-dependence using the finite Fourier transform. The radial $(r)$ dependence will be expanded using the finite Fourier-Bessel tranşform or, in the notation of Watson, 16 the Dini transform. The conditions that must be imposed on the functions in order for the transforms to exist vary with the transform. The conditions for the four mentioned transforms are given by Sneddon ${ }^{17}$ on pp. 237, 266, 309, and 314, respectively. These conditions cover a wide range of functions and except for the Laplace tran'sform apply to any physical situation. Backus 18 has examined the conditions for the validity of the Laplace transform in the one-dimensional plasma oscillation problem and has found a wide range of conditions for the transform to exist. It appears reasonable to assume its validity in our $\mathrm{N}$-stream, finite-geometry problem also.

Following then, e.g. , Sneddon, ${ }^{17}$ we obtain for the transforms $\left\{\begin{array}{l}\mathrm{f}_{1}^{\lambda \mathrm{km} \omega} \\ \phi_{1}^{\lambda \mathrm{km} \omega} \\ \mathrm{A}_{1}^{\lambda \mathrm{km} \omega} \\ \dot{p}_{\mathrm{z}}^{1 \lambda \mathrm{km} \omega}\end{array}\right\}=\int_{0}^{\infty} \mathrm{dt} \int_{-\infty}^{\infty} \mathrm{dz} \int_{0}^{2 \pi} \mathrm{d} \theta \int_{0}^{\mathrm{a}} \mathrm{r} \mathrm{dr}\left\{\begin{array}{c}\mathrm{f}_{1} \\ \hat{\phi} \\ \hat{\AA} \\ \dot{p}_{\mathrm{z}}\end{array}\right\} J_{\mathrm{m}}\left(\lambda_{\mathrm{j}} \mathrm{r}\right) \mathrm{e}^{-i m \theta-i k z+i \omega t}$ The quantities $\hat{\phi}, \hat{A}$ are defined by $\hat{\phi}=\phi_{1}, \hat{A}=A_{1}$ for $r \leq a$, and are defined only on this range of $r$. Under the above conditions, the following inversion theorem holds 


$$
\left\{\begin{array}{c}
f_{1} \\
\hat{\phi}_{1} \\
\hat{A}_{1} \\
\dot{p}_{z}^{1}
\end{array}\right\}=\sum_{m=-\infty}^{\infty} \sum_{j=1}^{\infty} b_{j} \int_{-\infty}^{\infty} d k \int_{W}^{d \omega}\left\{\begin{array}{l}
f_{1}^{\lambda k m \omega} \\
\phi_{1}^{\lambda k m \omega} \\
A_{1}^{\lambda k m \omega} \\
\dot{p}_{z}^{\lambda k m \omega}
\end{array}\right\} \frac{J_{m}\left(\lambda_{j} r\right)}{(2 \pi)^{3}} e^{i m \theta+i k z-i \omega t}
$$

Since the improper integrals in Eq. (35) converge uniformly, they may be interchanged, implying also that the integrals and sums in Eq. (36) may be freely interchanged, so that Eq. (36) is unique. We turn now to a definition of the symbols in Eq. (36). The quantities $m$ are the real integers. The coefficients $b_{j}$ are defined as

$$
b_{j}=\frac{2}{a^{2}} \frac{1}{\left[J_{m}\left(\lambda_{j} a\right)\right]^{2}} \frac{\lambda_{j}^{2}}{h^{2}+\lambda_{j}^{2}-m^{2} / a^{2}} .
$$

These coefficients are given by Sneddon ${ }^{17}$ (p. 315 ) or by Watson ${ }^{16}$ (p.597). The $\lambda_{j}$ are the discrete positive solutions of

$$
\lambda_{j} J_{m}^{\prime}\left(\lambda_{j} a\right)+h J_{m}\left(\lambda_{j} a\right)=0
$$

where $J_{m}^{\prime}(z)=\frac{d}{d z}\left[J_{m}(z)\right]$. In the proof of the Fourier-Bessel (Dini) expansion by Watson, ${ }^{16}$ the quantity $h$ is assumed real and $m \geq 0$. Watson points out that if ha $+m \leq 0$, an additional term appears in the Dini expansion. We will see later that for our choice of $h$, this can never occur.

Careful review of Watson's ${ }^{19}$ derivation shows that most of the above results are true also if $\mathrm{h}$ in Eq. (37) is complex. There are two modifications. One is that only the discrete $\lambda_{j}$, the solution of Eq. (38), that satisfy

$$
\operatorname{Re}\left(\lambda_{j}\right)>0
$$


are used in the expansion of Eqs. (35) and (36). The other modification occurs if the denominator of Eq. (37) is zero. This may occur only for complex $\lambda_{j}$. In fact, it is easy to show that for a given $h$, this may occur for only one $\dot{\lambda}_{i}$. For this case Watson's ${ }^{16}$ proof for the expansion may be generalized with the result that $b_{j}$ is now

$$
b_{j}=\frac{2}{a^{2}\left[J_{m}\left(\lambda_{j} a\right)\right]^{2}}\left(a \lambda_{j}^{2} \frac{d}{d \lambda_{j}}+\frac{4}{3}(1+h a)\right):
$$

(A detailed proof will be published later.) The term $\frac{d}{d \lambda_{j}}$ is a shorthand notation. It means differentiation with respect to $\lambda_{j}$ of both terms, $f_{1}^{\lambda k m \omega}$ (or $\phi_{1}^{\lambda k m \omega}$, etc. ) and $J_{m}\left(\lambda_{j} r\right)$, in Eq. (36). The term $f_{1}^{\lambda k m \omega}$ (or $\phi_{1}^{\lambda k m \omega}$, etc.) is to be considered a function of $\lambda_{j}$ only through its dependence on $J_{m}\left(\lambda_{j} r\right)$ in Eq. (35). Further discussion of this singular case follows below after Eq. (154). The Dini expansion is evidently valid also for $m$ negative. One simply writes $J_{m}(z)=(-1)^{m_{J}}(z)$ in Eq. (36).

If the function $f(s)$ is bounded and continuous the Dini series converges uniformly to the value of the function at both ends, $r=0$ and $r=a$. Since this may appear surprising, we present the argument. Consider first the point $r=0$. We see from the expansion, Eq. (36), that for each $m=0$, the radial sum is zero, while for $m=0$, it is some constant (because $\left.J_{0}(0) \nsucc 0, J_{m}(0)=0\right)$. However, a physical function that behaves as $e^{\operatorname{im} \theta}(\mathrm{m}=0)$ must be zero at the origin if the function is spatially continuous. Only if $\mathrm{m}=0$ can the function be nonzero at the origin. Hence the convergence is continuous at $r=0$.

At $r=a$, Watson $^{16}$ (p. 604 and footnote p. 605). states clearly that the Dini series converges uniformly. This is not true for the Fourier-Bessel series which converges uniformly only if $f(s)=0$ at $r=a$. These series differ in that the latter is obtained by setting $h=\infty$ in Eq. (38). The discussion 
following Eq. (73) shows that this cannot occur. The difference between the two expansions is interesting and important.

We note again that $h$ may be arbitrary. Different h's simply define different sets of expansion vectors $\lambda_{j}$, through Eq. (38).

Turning now to the $\mathbf{k}$ integral in Eq. (36), we note that the path of integration is along the real axis, as is usual in Fourier transforms. In Eq. (35) too, $k$ is assumed real. For the Laplace transform part of Eq. (36), the contour $W$ is parallel to the real axis and is above all singularities of the integrand (see Fig. 2) viewed as functions of complex $\omega$.

Having given the definitions and explanations of the various terms in Eq. (36), we shall now proceed to apply the above results. We shall insert the expression in Eq. (36) into the right-hand side of Eqs. (31) and (33) and carry out the spatial integration. By a proper choice of orientation of our vector space coordinate system, i. e., by a suitable set of $\lambda_{j}$ obtained by a proper choice of $h$ in Eq. (38), we will obtain the transforms of $\phi_{1}$ and $A_{1}$ in terms of the transforms of $f_{1}$.

In order to simplify the expressions we shall adopt the following definitions:

$$
\left.\begin{array}{c}
\mathrm{f}_{1}^{\lambda \mathrm{km} \omega} \\
\phi_{1}^{\lambda \mathrm{km} \omega} \\
\mathrm{A}_{1}^{\lambda \mathrm{km} \omega} \\
\dot{\mathrm{p}}_{\mathrm{z}}^{\lambda \mathrm{km} \omega}
\end{array}\right\} \equiv\left\{\begin{array}{l}
\mathrm{f}^{\tau} \\
\phi^{\tau} \\
\mathrm{A}^{\tau} \\
\dot{\mathrm{p}}^{\tau}
\end{array}\right.
$$

and 


$$
\sum \sum \iint \equiv \sum_{m=-\infty}^{\infty} \sum_{j=1}^{\infty} b_{j} \int_{-\infty}^{\infty} d k \int_{W} d \omega
$$

Inserting Eq. (36) into the right-hand side of Eq. (31), now gives (including the retardation term)

$\phi_{1}\left(\underset{\sim}{r^{\prime}}, t\right)=e \int\left[\sum \sum \iint \frac{f^{\tau} J_{m}\left(\lambda_{j} r\right) e^{i m \theta+i k z-i \omega\left(t-\left|\underset{\sim}{r}-\underline{\sim}^{\prime}\right| / / c\right)}}{(2 \pi)^{3}|\underset{\sim}{r}-\underset{\sim}{r}|}\right] r d r d \theta d z d p$.

If the sums and integrals are uniformly convergent, then the spatial integration and the other sums and integrations may be interchanged, so that the spatial integrations may be performed first. If $\phi(r, \theta, z)$ are continuous and bounded, then the Fourier series, ${ }^{20}$ Dini $^{21}$ (Fourier-Bessel) series, and Fourier integral ${ }^{22}$ are uniformly convergent. The Laplace transform may be regarded as the Fourier transform of a function equal to $\phi(t) e^{-c t}$ for $t>0$ and equal to zero for $t<0$. By the Fourier theorems, ${ }^{22}$ this function, regarded as a function of $\omega$, is uniformly convergent except at $t=0$. Hence the interchange of integrals in Eq. (43) fails at $|\underline{\sim}-\underset{\sim}{r}|=c t$, and gives a different though finite value at this point. However, this means that only on this surface, which gives an almost zero contribution to the volume integral, does the integral interchange give a different result. Hence Eq. (44) still follows from Eq. (43) if $\phi(r, \theta, z, t) e^{-c t}$ is bounded and continuous.

Equation (43) then becomes

$$
\phi_{1}\left(\underline{r}^{\prime}, t\right)=\frac{e}{(2 \pi)^{2}} \sum \sum \iint\left[I_{s}\right] \int f^{\tau} d p
$$


where

$I_{s}=\int_{0}^{a} r d r \int_{-\infty}^{\infty} d z \int_{0}^{2 \pi} d \theta \frac{J_{m}(\lambda r) e^{\left.i m \theta+i k z-i \omega|t-| \underset{\sim}{r}-\underset{\sim}{r^{\prime}} \mid / c\right)}}{\mid \underset{\sim}{r}-\underset{\sim}{r^{\prime} \mid}}$.

The radial integration limit, a, is fixed by the fact that the magnetic field constrains the particles to remain within the cylinder. Equation (33) may be written in a similar form. The radial integration limit there too is a, since $f_{1}$ and $f_{0}$ are zero for $r \geq a$.' With the notation of Eq! (44): we have

$A_{1}\left(r^{\prime}, t\right)=\frac{e}{(2 \pi)^{3}} \sum \sum \iint\left[I_{s}\right] \int\left(v f^{\tau}-\frac{e A^{\tau} f_{0}}{c \gamma^{3} m_{0}}\right) d p$

Our next step is, then, to evaluate the integral $I_{s}$, which will be explicitly done in the following section.

\subsection{Integration of the Spatial Integral, $\mathrm{I}_{\mathrm{S}}$}

The integral which we shall evaluate explicitly is

$I_{s}=\int_{0}^{a} r d r \int_{-\infty}^{\infty} d z \int_{0}^{2 \pi} d \theta \frac{J_{m}(\lambda r) \exp \left\{i m \theta+i k z-i \omega t+\frac{i \omega}{c}\left[r^{2}+r^{\prime 2}-2 r r^{\prime} \cos \left(\theta-\theta^{\prime}\right)+\left(z-z^{\prime}\right)^{2}\right] 1 / 2\right\}}{\left[r^{2}+r^{\prime 2}-2 r r^{\prime} \cos \left(\theta-\theta^{\prime}\right)+\left(z-z^{\prime}\right)^{2}\right]^{1 / 2}}$

The first step is to make the change of variable, $\theta^{\prime \prime}=\theta-\theta^{\prime}, z^{\prime \prime}=z-z^{\prime}$. Equation (46) then becomes

$$
I_{s}=I_{0} e^{i m \theta^{\prime}+i k z^{\prime}-i \omega t}
$$

where, 
$I_{0}=\int_{0}^{a} r d r \int_{-\infty}^{\infty} d z^{\prime \prime} \int_{\theta^{\prime}}^{2 \pi-\theta^{\prime}} \frac{d \theta^{\prime \prime} J_{m}(\lambda r) \exp \left[i m \theta^{\prime \prime}+i k z^{\prime \prime}+\frac{i \omega}{c}\left(R^{2}+z^{\prime \prime}\right)^{1 / 2}\right.}{\left(R^{2}+z^{\prime \prime 2}\right)^{1 / 2}}$

and we have set

$$
R^{2} \equiv r^{2}+r^{\prime 2}-2 r r^{\prime} \cos \theta^{\prime \prime}
$$

For the $z$ integral various tables give the result

$I_{r \theta z}=\int_{-\infty}^{\infty} \frac{\exp \left[-i k z^{\prime \prime}+\frac{i \omega}{c}\left(R^{2}+z^{\prime \prime 2}\right)^{1 / 2}\right]}{\left(R^{2}+z^{\prime \prime 2}\right)^{1 / 2}} d z^{\prime \prime}=2 K_{0}\left\{k R\left[1-\left(\frac{\omega}{k c}\right)^{2}\right]^{1 / 2}\right\}$

where $\mathrm{K}_{0}$ is the Bessel function of imaginary argument. Campbell and Foster 23,24 give for the range of validity of this integral $\operatorname{Im}(\omega) \geq 0$. Other tables 25 give $\operatorname{Im}(\omega)>0$. To resolve the differences, we have derived the above result, which indicates that Eq. (50) is valid for

$$
\begin{aligned}
& \operatorname{Im}(\omega) \geq 0 \quad \text { if } \quad\left|\operatorname{Re}\left(\frac{\omega}{\mathrm{kc}}\right)\right|<1, \\
& \operatorname{Im}(\omega)>0 \quad \text { if } \quad\left|\operatorname{Re}\left(\frac{\omega}{\mathrm{kc}}\right)\right|>1 .
\end{aligned}
$$

The phase of the argument of $\ddot{K}_{0}$ is given by

$$
-\frac{\pi}{2}<\arg \left(\mathrm{k}^{2}-\frac{\omega^{2}}{\mathrm{c}^{2}}\right)^{1 / 2}<\frac{\pi}{2} \text {. }
$$

For other phases of the argument, the integral, Eq. (50), diverges including when $\operatorname{Im}(\omega)=0$, and $|\operatorname{Re}(\omega / \mathrm{kc})|>1$. For the above results $R>0$ is required, which is evidently satisfied as $\left|r-r^{\prime}\right|>0\left(\left|r-r^{\prime}\right|^{2}=R^{2}+z^{\prime \prime 2}\right)$ is required for the validity of the retarded potentials formulae in Eqs. (16) and (17). 
Our next step is to perform the $\theta^{\prime \prime}$ integration in Eq. (48). Thus, we now desire to evaluate

$$
I_{r \theta}=\int_{-\theta^{\prime}}^{2 \pi-\theta^{\prime}} e^{i m \theta^{\prime \prime}} \cdot 2 K_{0}\left[R\left(k^{2}-\omega^{2} / c^{2}\right)^{1 / 2}\right] d \theta^{\prime \prime}
$$

where $\mathrm{R}$ is given by Eq. (48). The integration is facilitated by using a Bessel function addition theorem tabulated by Erdelyi, ${ }^{26}$ and essentially derived by Stratton. ${ }^{27}$ The theorem is

$$
K_{\nu}(w) e^{i \nu \psi}=\sum_{n=-\infty}^{\infty} K_{n+\nu}(z) I_{n}(z) e^{i n \theta}
$$

where

$$
\left.\begin{array}{rl}
w^{2} & =z^{2}+z^{2}-2 z z \cos \theta \\
|z| & <|z|, \text { and } \\
w e^{i \psi} & =z-z e^{-i \theta} \text { defines } \psi
\end{array}\right\}
$$

For the case where $\nu=0$, and using our parameters, i. e., $\theta \rightarrow \theta^{\prime \prime}$, $w^{2} \rightarrow\left(k^{2}-\omega^{2} / c^{2}\right)\left(r^{2}+r^{\prime 2}-2 r r^{\prime} \cos \theta^{\prime \prime}\right)$, we obtain from Eq. (54)

$$
K_{0}(\kappa R)=\sum_{n=-\infty}^{\infty} K_{n}(\kappa r) I_{n}\left(\kappa r^{\prime}\right) e^{i n \theta^{\prime \prime}}
$$

valid for $r^{\prime}<r$. We have also defined

$$
\kappa=\left(\mathrm{k}^{2}-\omega^{2} / \mathrm{c}^{2}\right)^{1 / 2} ;-\frac{\pi}{2}<\arg \kappa<\frac{\pi}{2}
$$

using Eq. (52). Inserting Eq. (56) into Eq. (53), the $\theta^{\prime \prime}$ integral becomes

$$
\int_{-\theta^{\prime}}^{2 \pi-\theta^{\prime}} e^{i m \theta^{\prime \prime}+i n \theta^{\prime \prime}} d \theta^{\prime \prime}= \begin{cases}2 \pi & \text { for } n=-m \\ 0 & \text { for } n \neq m\end{cases}
$$


Thus all the sums of Eq. (56) give zero, except the one where $n=-m$. (The radial integrals remain finite; or more accurately, we see that although $\mathrm{K}_{\mathbf{n}}$ $(\kappa r) \rightarrow \infty$ as $r \rightarrow 0$, the integral must be broken up into two parts, one valid for $r>r^{\prime}$, the other for $r<r^{\prime}$, so that $K_{n}(\kappa r) I_{n}\left(\kappa r^{\prime}\right)$ tends to a finite value as $r \rightarrow 0$, because $r^{\prime}<r$. For the case $r^{\prime}>r$, we have $I_{n}(\kappa r)$ in the integrand, which approaches zero as $r \rightarrow 0$ and hence here too the integrand remains finite.)

We note too that although the point $r=r^{\prime}$ has been left out in the expansion, Eq. (56), this is unimportant since $K_{0}(k R)$ is continuous at all $r$ and $\theta "$, except the point $R=0$, which has been left out of the integral by the definitions of the retarded potentials.

Using now the well-known result $K_{-m}(z)=K_{m}(z), I_{-m}(z)=I_{m}(z)$, and Eqs. (58), (53), and (56), we obtain

$$
I_{r \theta}=\left\{\begin{array}{lll}
4 \pi\left[K_{m}(k r) I_{m}\left(k r^{\prime}\right)\right] & \text { when } & r^{\prime}<r \\
4 \pi\left[K_{m}\left(\kappa r^{\prime}\right) \operatorname{Im}(k r)\right] \text { when } & r^{\prime}>r .
\end{array}\right.
$$

We may now carry out the radial integration indicated in Eq. (48). Let us first consider the case where ' $r$ '>a. Then,

$$
\begin{aligned}
I_{0} & =\int_{0}^{a} I_{r \theta} J_{m}(\lambda r) r d r \\
& =4 \pi K_{m}\left(\kappa r^{\prime}\right) \int_{0}^{r^{\prime}} I_{m}(\kappa r) J_{m}(\lambda r) r d r+4 \pi I_{m}\left(k r^{\prime}\right) \int_{r^{\prime}}^{a} K_{m}(\kappa r) J_{m}(\lambda r) r d r
\end{aligned}
$$

These integrals are easily evaluated using formulae (194) and (232) of McLachlan. ${ }^{28}$ After cancelling four terms, the result is

$$
\frac{I_{0}}{4 \pi}=\frac{J_{m}\left(\lambda r^{\prime}\right) \kappa r^{\prime}}{\lambda^{2}+\kappa^{2}}\left[K_{m}\left(\kappa r^{\prime}\right) I_{m+1}\left(\kappa r^{\prime}\right)+K_{m+1}\left(\kappa r^{\prime}\right) I_{m}\left(\kappa r^{\prime}\right)\right]
$$




$$
+\frac{I_{m}\left(\kappa r^{\prime}\right)}{\lambda^{2}+\kappa^{2}}\left[\lambda a K_{m}(\kappa a) J_{m+1}(\lambda a)-\kappa a J_{m}(\lambda a) K_{m+1}(\kappa a)\right]
$$

This result may be simplified using the identity, Eq. (219) of McLachlan, ${ }^{28}$

$$
I_{\nu}(z) K_{\nu}^{\prime}(z)-I_{\nu}^{\prime}(z) K_{\nu}(z)=-1 / z
$$

This identity may be rewritten using formulae (164) and (209) of McLachlan, 28

$$
\begin{aligned}
\mathrm{I}_{\nu}^{\prime} & =\frac{\nu}{\mathrm{z}} \mathrm{I}_{\nu}+\mathrm{I}_{\nu+1}, \\
\mathrm{~K}_{\nu}{ }^{\prime} & =\frac{\nu}{\mathrm{z}} \mathrm{K}_{\nu}-\mathrm{K}_{\nu+1},
\end{aligned}
$$

to obtain

$$
\mathrm{I}_{\nu} \mathrm{K}_{\nu+1}+\mathrm{I}_{\nu+1} \mathrm{~K}_{\nu}=\frac{1}{\mathrm{z}}
$$

This result allows us to simplify Eq. (61). We note that the expression in the second bracket is a constant. Thus we obtain from Eq. (61)

$$
I_{0}=4 \pi\left[\frac{J_{m}\left(\lambda r^{\prime}\right)}{\lambda^{2}+\kappa^{2}}+\frac{I_{m}\left(\kappa r^{\prime}\right)}{\lambda^{2}+\kappa^{2}} \stackrel{\hat{T}}{\wedge}\right] \quad\left(r^{\prime}<a\right)
$$

where

$$
\hat{\mathrm{T}}=\lambda a \mathrm{~K}_{\mathrm{m}}(\kappa a) J_{\mathrm{m}+1}(\lambda a)-\kappa a \mathrm{~J}_{\mathrm{m}}(\lambda a) \mathrm{K}_{\mathrm{m}+1}(\kappa a)
$$

The insertion of this result into Eq. (47) now gives the explicit evaluation of Eq. (46) valid for $r^{\prime}<a$.

Having evaluated $I_{S}$ for $r^{\prime}<a$, we turn now to the task of evaluating. this integral when $r^{\prime}>a$. The results of this section remain the same up to Eq. (60), which now becomes

$$
\begin{aligned}
I_{0} & =\int_{0}^{a} I_{r \theta} J_{m}(\lambda r) r d r \\
& =4 \pi K_{m}\left(\kappa r^{\prime}\right) \int_{0}^{a} I_{m}(k r) J_{m}(\lambda r) r d r
\end{aligned}
$$


because since $r^{\prime}>a, r^{\prime}>r$ and hence only the second of Eqs. (59) should be used to evaluate this integral. Using formulae (194) of McLachlan, ${ }^{28}$ we can evaluate this integral explicitly. We obtain

$$
\begin{aligned}
I_{0} & =\frac{4 \pi K_{m}\left(\kappa r^{\prime}\right)}{\lambda^{2}+\kappa^{2}}\left[\kappa a I_{m+1}(\kappa a) J_{m}(\lambda a)+\lambda a J_{m+1}(\lambda a) I_{m}(k a)\right] \\
& =\frac{4 \pi K_{m}\left(k r^{\prime}\right)}{\lambda^{2}+\kappa^{2}} \tilde{T}\left(r^{\prime}>a\right)
\end{aligned}
$$

which defines $\tilde{T}$. Insertion of this result into Eq. (47) now gives the explicit evaluation of $I_{s}$. This result, together with Eq. (65), now gives $I_{s}$ valid for all values of $r^{\prime}$. Although strictly the result is undefined for $r^{\prime}=a$, it is easily verified that Eqs, (65) and (68) give the same result at $r^{\prime}=a$, so that there is no difficulty.

Having evaluated $I_{S}$, we turn now to the potentials $\phi$ and $A$ given by Eqs. (44) and (45) and the task of obtaining the transforms of $\phi$ and $A$ in terms of the transforms $f^{\tau}$ of $f$.

\subsection{Evaluation of the Transforms $\phi^{\top}$ and $A^{\tau}$}

With the above results for $I_{S}$, Eqs. (65) and (47), inserted into Eq. (44) for $\phi$, we now have

$$
\hat{\phi} \equiv \phi_{1}=\frac{4 \pi \mathrm{e}}{(2 \pi)^{3}} \sum \sum \iint \frac{\mathrm{e}^{i m \theta^{\prime}+\mathrm{ikz}-i \omega t}}{\lambda^{2}+\kappa^{2}}\left\{J_{m}\left(\lambda \mathrm{r}^{\prime}\right)+\hat{\mathrm{TI}} \mathrm{m}^{\left(\kappa \mathrm{r}^{\prime}\right)}\right\} \underset{\text { for } \mathrm{r}^{\prime} \leq a}{\int \mathrm{f}^{\top} \mathrm{dp}}
$$

To obtain the corresponding result for $r^{\prime} \geq a$, we insert Eqs. (68) and (47) into Eq. (44), giving 


$$
\tilde{\phi} \equiv \phi_{1}=\frac{4 \pi \mathrm{e}}{(2 \pi)^{3}} \sum \sum \iint \frac{\mathrm{e}^{\mathrm{im} \theta^{\prime}+\mathrm{ikz} z^{\prime}-i \omega t}}{\lambda^{2}+\kappa^{2}}\left[\mathrm{~K}_{\mathrm{m}}\left(\kappa \mathrm{r}^{\prime}\right) \tilde{\mathrm{T}}\right] \int \mathrm{f}^{\tau} \mathrm{dp}
$$

It is easily verified that $\hat{\phi}\left(r^{\prime}=a\right)=\tilde{\phi}\left(r^{\prime}=a\right)$ by examining the bracket terms in Eqs. (69) and (70) and using the explicit formulae for $\hat{\mathrm{T}}$ and $\tilde{\mathrm{T}}$. Similarly

$$
\frac{\partial \hat{\phi}}{\partial r}\left(r^{\prime}=a\right)=\frac{\partial \tilde{\phi}}{\partial r}\left(r^{\prime}=a\right)
$$

may be verified.

There are evidently two similar expressions for the vector potential. Inserting Eqs. (47) and (65) into Eq. (45), and then Eqs. (47) and (68), into Eq. (45), results in the following two equations for the different ranges of $r^{\prime}$ :

$$
\begin{gathered}
\hat{A} \equiv A_{1}=\frac{4 \pi e}{(2 \pi)^{3} \cdot c} \sum \sum \iint \frac{e^{i m \theta^{\prime}+i k z^{\prime}-i \omega t}}{\lambda^{2}+\kappa^{2}}\left[J_{m}\left(\lambda r^{\prime}\right)+\hat{T}_{m}\left(\kappa r^{\prime}\right)\right] \int\left(v f^{\tau}-\frac{e A^{\tau} f_{0}}{c \gamma^{3} m_{0}}\right) d p, \\
\text { for } r^{\prime} \leq a,
\end{gathered}
$$

and

$$
\begin{aligned}
& \tilde{A} \equiv A_{1}=\frac{4 \pi \mathrm{e}}{(2 \pi)^{3} \mathrm{c}} \sum \sum \iint \frac{e^{i m \theta^{\prime}+\mathrm{ikz} z^{\prime}-\mathrm{i} \omega t}}{\lambda^{2}+\kappa^{2}}\left[\mathrm{~K}_{\mathrm{m}}\left(\kappa \mathrm{r}^{\prime}\right) \tilde{\mathrm{T}}\right] \int\left(v f^{\tau}-\frac{e \mathrm{~A}^{\tau_{\mathrm{f}}}}{\mathrm{c} \gamma^{3} \mathrm{~m}_{0}}\right) \mathrm{dp}, \\
& \text { for } r^{\prime} \geq a \text {. }
\end{aligned}
$$

Equations (69) and (71) closely resemble the inverse transform expressions of Eq. (36). They differ in the additional term in the bracket $\hat{\mathrm{TI}}_{\mathrm{m}}\left(\kappa \mathrm{r}^{\prime}\right)$. We have, however, a degree of freedom in the choice of the expansion vectors $\lambda_{j}$, provided by the choice in $h$ of Eq. (38). This will allow us to set $\hat{T}=0$ in Eqs. (69) and (71). We shall now show this in detail. 
Let us set, in Eq. (38),

$$
h=-k \frac{K_{m}^{\prime}(k a)}{K_{m}(k a)} .
$$

This quantity $h$ is always finite because $K_{m}(\kappa a)$ has no zeros in the right half of the complex plane, ${ }^{29}$ which by Eqs. (51) and (57) is the domain of definition of this function.

We note too that $h$ can never be negative. If $h$ were negative, then for example let ha $=-B$, where $B>0$ is some number. Then Eq. (73) may be put in the form $\mathrm{BK}_{\mathrm{m}}(\kappa a)-\kappa a K_{\mathrm{m}}^{\prime}(\kappa a)=0$. However, by Erdelyi, ${ }^{26}$ bottom of p. 62 , this expression has no zeros for $k a$ in the right half of the complex plane. Since by Eqs. (51) and (57) this is precisely the domain of definition of $k a$, we see that the above expression cannot be zero and hence $h$ cannot be negative. Hence $($ ha $+m$ ) also cannot be negative. (Recall $\mathrm{m}>0$ !)

Consider next whether (ha $+m$ ) can equal zero. Since $h$ can never be negative, we must first have $m=0$. By Eq. (73) then

$$
h_{\mathrm{ha}}=-\frac{\kappa a \mathrm{~K}_{1}(\kappa a)}{\mathrm{K}_{0}(\kappa a)}
$$

Since $K_{1}(\kappa a)$ has no zeros, we see that ha $=0$ only when $\kappa a=0$, i. e., when $\omega=\mathrm{kc}$. . However, since this is a branch point in the complex $\omega$-plane and, as will be seen later, is left out of the domain of integration, we see that in the domain of interest ha $+m=0$, and in fact ha $+m \leq 0$ cannot occur. Hence the additional term in the Dini expansion, Eq. (36), does not occur. This point was mentioned earlier, in the discussion following Eq. (38). 
Equation (38) now becomes

$$
\lambda_{j} J_{m}^{\prime}\left(\lambda_{j} a\right) K_{m}(k a)-k J_{m}\left(\lambda_{j} a\right) K_{m}^{\prime}(k a) \equiv 0 .
$$

Using the formulae (25) and (209) of McLachlan, ${ }^{28}$ this may be written as

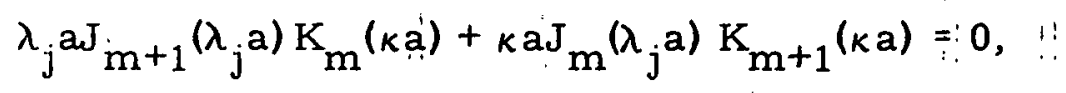

which is identical to $\hat{T}=0$. Hence with the choice of the coordinate vectors $\lambda_{j}$ for the expansion in Eqs. (69)-(72), defined by Eq. (75), we obtain automatically $\hat{T}=0$. Equations (69) and (71) are now of the form of Eq. (36). Since the expansion is unique we obtain automatically the transforms $\phi^{\tau}$ and $A^{\tau}$ from Eqs. (69) and (71),

$$
\phi^{\tau}=\frac{4 \pi e}{\lambda^{2}+\kappa^{2}} \int f_{11}^{\tau} d p
$$

and

$$
\mathrm{A}^{\tau}=\frac{4 \pi \mathrm{e}}{\mathrm{c}\left(\lambda^{2}+\kappa^{2}\right)} \int\left(\mathrm{vf} \tau-\frac{\mathrm{eA}^{\tau} \mathrm{f}_{0}}{\mathrm{c} \gamma^{3} \mathrm{~m}_{0}}\right) \mathrm{dp} .
$$

Equations (77) may be simplified. By Eqs. (11) and (27), $\int f_{0} d p=n_{0}$, so that

$$
\int-\frac{e A^{\tau} f_{0}}{c \gamma^{3} m_{0}} d p=-\frac{e A^{\tau} n_{0}}{c \gamma^{3} m_{0}}
$$

and Eq. (77) may be written as

$$
A^{\tau}=\left(1+\frac{4 \pi e^{2} n_{0}}{\gamma^{3} m_{0} c^{2}} \frac{1}{\lambda^{2}+\kappa^{2}}\right)^{-1} \cdot \frac{4 \pi e}{c\left(\lambda^{2}+\kappa^{2}\right)} \int v f^{\tau} d p
$$


To simplify the notation we shall adopt the definitions

$$
\nu \equiv \frac{\dot{e}^{2}}{\gamma^{3} m_{0} c^{2}} n_{0} \pi a^{2}, \quad g=\frac{4 \pi}{\pi a^{2}\left(\lambda^{2}+k^{2}\right)}
$$

These quantities are dimensionless. When $\mathbf{m}_{0}$ is the electron mass, and $\gamma=1, \nu$ gives the number of électrons in the plasma cylindè in a disk of length equal to the classical electron radius. The quantity $g$ is a dimensionless quantity depending on the system geometry, i. e., it depends on the plasma cylinder radius and the wave lengths. With these quantities, Eqs. (76) and (79) become

$$
\phi^{\tau}=\mathrm{e} \pi \mathrm{a}^{2} \mathrm{~g} \int \mathrm{f}^{\tau} \mathrm{dp}
$$

and

$$
\mathrm{A}^{\tau}=\frac{\mathrm{e} \pi \mathrm{a}^{2} \mathrm{~g}}{1+\nu \mathrm{g}} \int_{\mathrm{i}}^{\mathrm{v}} \mathrm{f}^{\tau} \mathrm{dp}
$$

Equations (81) and (82) now give the desired transformis. Since the $\lambda_{j}$ in . Eqs. (81) and (82) are now functions of $\mathrm{m}, \mathrm{k}$, and $\omega$ through Eq. (75), it is clear that in Eq. (36) or Eqs. (69) and (71), the summation over the $\lambda_{j}$ must be performed first and then the summation and two integrations. We note also that Eqs. (70) and (72) differ from Eqs. (69) and (71) only in the radial depen-dence. If the disturbance in the plasma cylinder is set up so that only one mode, or one set of $\lambda_{j}, m, k$, and $\omega$, is excited, then for the potential $\phi$ or A within the cylinder

$$
\left\{\begin{array}{l}
\phi \\
\mathrm{A}
\end{array}\right\}=\left\{\begin{array}{l}
\phi^{\tau} \\
\mathrm{A}^{\tau}
\end{array}\right\} \quad \mathrm{e}^{\mathrm{im} \theta+i k z-i \omega t} \mathrm{~J}_{\mathrm{m}}\left(\lambda_{\mathrm{j}} \mathrm{r}\right), \quad \mathrm{r}<\mathrm{a}
$$


while outside the cylinder,

$$
\left\{\begin{array}{l}
\phi \\
\dot{A}
\end{array}\right\}=\left\{\begin{array}{l}
\phi^{\tau} \\
A^{\tau}
\end{array}\right\} \cdot e^{i m \theta+i k z-i \omega t_{k}} K_{m}\left(k r^{\prime}\right) \tilde{T}, r>\dot{d} .
$$

By the earliẹr discussion these quantities and their derivatives are continuous at $r=a$.

Having obtained the transforms $\phi^{\tau}$ and $A^{T}$, Eqs. (81) and (82), as functions of $\mathrm{f}^{\tau}$, we turn now to the kinetic equation, Eq. (28), and the momentum equation, Eqs. (30). We shall take the transforms of these two equations, thereby obtaining (from the kinetic equation) $\mathrm{f}^{\boldsymbol{\tau}}$ in terms of $\dot{\mathrm{p}}^{\boldsymbol{\tau}}$ and the initial conditions and (from the momentum equation) $\dot{\mathrm{p}}^{\boldsymbol{T}}$ in terms of $\phi^{\tau}$ and $\mathrm{A}^{\boldsymbol{T}}$, so that we can solve for the transforms in terms of the initial conditions and thereby solve the problem.

In the next section we shall obtain the transforms of the kinetic and momentum equations. These steps are a generalization of Jackson' ${ }^{2}$ procedure.

\subsection{Transforms of the Kinetic and Momentum Equations}

First we shall obtain the transform of the kinetic equation. We multiply Eq. (28) by $\mathrm{e}^{-\mathrm{im} \theta-\mathrm{ikz+i \omega t}} \mathrm{J}_{\mathrm{m}}(\lambda \mathrm{r})$ and integrate over $\mathrm{r} d \mathrm{~d} \mathrm{~d} \theta \mathrm{dz} \mathrm{dt}$. After integrating by parts with respect to $t$ and $z$, we obtain

$$
\begin{aligned}
& \int_{0}^{a} J_{m}(\lambda r) r d r \int_{0}^{2 \pi} e^{-i m \theta} d \theta\left\{\int_{-\infty}^{\infty} d z\left[f_{1} e^{-i k z+i \omega t}\right]_{0}^{\infty}\right. \\
&-i\left(\omega-\mathrm{kz}_{0}\right) \int_{-\infty}^{\infty} d z \int_{0}^{\infty} d t f_{1} e^{-i k z+i \omega t} \\
&\left.\quad+\frac{\partial f_{0}}{\partial p} \int_{-\infty}^{\infty} d z \int_{0}^{\infty} d t \dot{p}_{z} e^{-i k z+i \omega t}\right\}=0
\end{aligned}
$$


With the assumption that $\omega$ lies sufficiently high in the complex upper halfplane, the first term vanishes at the upper limit, i.e., for $t \rightarrow \infty$. The remalnele of the term is the spatial transform of the indtial displacement, to be called S. Thus

$S(p, \lambda, m, k) \equiv \int_{0}^{a} J_{m}(\lambda r) r d r \int_{0}^{2 \pi} e^{-i m \theta} d \theta \int_{-\infty}^{\infty} e^{-i k z}: d z\left[f_{1}(t=0)\right]$.

We note too that $\dot{z}_{0}$ and $\partial f_{0} / \partial p$ are independent of $r, \theta, z$, and $t$, and hence may be taken out of all the integral signs of Eq. (84). Recalling the definitions of the transforms in Eq. (35), we may rewrite Eq. (84) as

$$
\text { : } \mathrm{S}-\mathrm{i}\left(\omega-\mathrm{k} \dot{\mathrm{z}}_{0}\right) \mathrm{f}^{\tau}+\frac{\partial \mathrm{f}_{0}}{\partial \mathrm{p}} \dot{\mathrm{p}}^{\tau}=0,
$$

or solving for $\mathrm{f}^{\tau}$,

$$
\mathrm{f}^{\tau}=\left(\dot{\mathrm{p}}^{\tau} \frac{\partial \mathrm{f}_{0}}{\partial \mathrm{p}}+\mathrm{S}\right) \frac{1}{\mathrm{i}\left(\omega-\mathrm{kz} \bar{z}_{0}\right)}
$$

This gives the transform $\mathrm{f}^{\tau}$ as a function of $\dot{\mathrm{p}}^{\boldsymbol{\tau}}$ and the initial conditions.

We turn next to the momentum equation, Eq. (30). We again multiply by $\mathrm{J}_{\mathrm{m}}(\lambda \mathrm{r}) \mathrm{e}^{-\mathrm{im} \theta-\mathrm{i} \mathrm{kz}+\mathrm{i} \omega \mathrm{t}}$ and integrate over $\mathrm{r} \mathrm{dr} \mathrm{d} \theta \mathrm{dz} d \mathrm{~d}$. Integrating the terms $\partial \phi / \partial z$ and $\partial A / \partial z$ by parts and using the definitions of the transforms in Eq. (35), we easily obtain

$$
\dot{\mathrm{p}}^{\tau}=\frac{\mathrm{e}}{\mathrm{c}} \dot{\mathrm{z}}_{0} \mathrm{ikA} \mathrm{A}^{\tau}-\mathrm{eik} \phi^{\tau}
$$

This is the desired relation.

It is now a simple matter to obtain the solution of the transforms in terms of the initial conditions. We detail the steps. One inserts Eqs. (81) and (82) for $A^{\tau}$ and $\phi^{\tau}$ into Eq. (87), to obtain

$$
\dot{\mathrm{p}}^{\tau}=i k \mathrm{e}_{\pi \mathrm{a}}^{2} \mathrm{~g}\left[\frac{\mathrm{z}_{\mathrm{l}}}{\mathrm{c}} \frac{1}{1+\nu \mathrm{g}} \int \frac{\dot{\mathrm{z}}_{\mathrm{f}^{\tau}}}{\mathrm{c}} \mathrm{dp}-\int \mathrm{f}^{\tau} \mathrm{dp}\right] .
$$


Next we insert Eq. (86) for $\mathrm{f}^{\tau}$ into Eq. (88) and obtain

$\dot{p}^{\tau}=e^{2} \pi a^{2} g\left[\frac{\dot{z}_{0}}{c} \frac{1}{1+\nu g} \int \frac{\dot{z}_{0}}{c} \frac{\left(\dot{p}^{\tau} \cdot \frac{\partial f_{0}}{\partial p}+s\right)}{\omega / k-\dot{z}_{0}} d p-\int \frac{\left(\dot{p}^{\tau} \frac{\partial f_{0}}{\partial p} \mathbf{g}\right)}{\omega / k-\dot{z}_{0}} d p\right]$,

an equation involving only the unknown $\dot{\mathrm{p}}^{\tau}$. The quantity $\dot{\mathrm{p}}_{i}^{\tau}$ is not a constant independent of $p$, because by Eq. (29), $\dot{z}_{0}=a+b p$ and is linear in p. Hence the simplest assumption for. $\dot{\mathrm{p}}^{\tau}{ }^{\text {, }}$ is

$$
\dot{\mathrm{p}}^{\tau}=\alpha+\beta \mathrm{p} .
$$

With these assumptions for $\dot{\mathrm{p}}^{\tau}$ inserted into Eq. (89), that equation may be written in the form

$$
\mathrm{F}_{1}+\mathrm{F}_{2} \mathrm{p}=0
$$

where $F_{1}$ and $F_{2}$ are independent of p. Since Eq. (91) holds for a range of $\mathrm{p}$, in particular also for $\mathrm{p}=0$, the solution is $\mathrm{F}_{1}=0, \mathrm{~F}_{2}=0$. This provides the two equations needed to obtain the two constants of Eq. $(90)$. We will not evaluate this equation explicitly, but will now turn our attention to the manybeam problem. In the next section we will generalize the above equations to many beams and write explicitly the analog of Eq. (91), which in the N-beam case gives $2 \mathrm{~N}$ equations.

\subsection{The N-Beam Equations}

It is comparatively straightforward to generalize the above results to $\mathrm{N}$ beams. Let us first consider the extensions of Eq. (81) and (82) for $\phi^{\tau}$ and $A^{\tau}$. There are now $\mathrm{N}$ beams and hence $\mathrm{N}$ distribution functions, $\mathrm{f}_{i}$. Examination of Eq. (76) shows that for $\phi^{T}$ we now have 


$$
\phi^{\tau}=\pi \mathrm{a}^{2} \mathrm{~g} \sum_{i}^{\mathrm{N}} \mathrm{e}_{\mathrm{i}} \int \cdot \mathrm{f}_{\mathrm{i}}^{\tau} \mathrm{dp} .
$$

Examination of Eq. (77) et seq. shows that for $A^{7}$ we have

$$
\mathrm{A}^{\tau}=\frac{\pi \mathrm{a}^{2} \mathrm{~g}}{\left(1+\mathrm{g} \sum_{i}^{\mathrm{N}} \nu_{i}\right)} \sum_{i}^{\mathrm{N}} \mathrm{e}_{\mathrm{i}} \int \frac{\mathrm{z}_{0 i}}{\mathrm{c}} \mathrm{f}_{\mathrm{i}}^{\tau} \mathrm{dp} .
$$

There are also $\mathrm{N}$ kinetic equations and $\mathrm{N}$ generalized force equations. Hence Eqs. (86) and (87) are easily generalized to

$$
f_{i}^{\tau}=\left(\dot{p}_{i} \frac{\partial f_{0 i}}{\partial p}+s_{i}\right) \frac{i}{i\left(\omega-k \dot{z}_{0 i}\right)}
$$

and

$$
\dot{p}_{i}^{\tau}=e_{i} \frac{\dot{z}_{0 i}}{c} i k A^{\tau}-e_{i} i k \phi^{\tau}
$$

Of course, the subscript $i$ is not to be confused with the imaginary $i$, for which $i^{2}=-1$.

The desired equations are now obtained by analogy with Eqs. (88) and (89). We first insert Eqs. (92) and (93) into each of Eqs. (95) to obtain $\dot{p}_{j}^{\tau}=i k \pi \cdot a^{2} g e_{j} \sum_{i}^{N} e_{i}\left[\frac{\dot{z}_{0 j}}{c} \frac{i}{1+g \sum_{i} \nu_{i}} \int \frac{\dot{z}_{0 i}}{c} f_{i}^{\tau} d p-\int f_{i}^{\tau} d p\right]$,

which gives $\mathrm{N}$ equations, one for each $\mathrm{j}$. Inserting the expression for $\mathrm{f}_{i}^{\top}$ given by Eq. (94) into Eq. (96) gives the desired equation which may be solved for the $\dot{p}_{j}^{\tau}$,

$\dot{p}_{j}^{\tau}=\pi a^{2} g e_{j} \sum_{i}^{N} e_{i}\left[\frac{\dot{z}_{0 j}}{c} \frac{1}{1+g \sum_{i} \nu_{i}} \int \frac{\dot{z}_{0 i}}{c} \frac{\left(\dot{p}_{i}^{\tau} \frac{\partial f_{0 i}}{\partial p}+s_{i}\right)}{\omega / k-\dot{z}_{0 i}} d p-\int \frac{\left(p_{i} \frac{\partial f_{0 i}}{\partial p}+S_{i}\right)}{\omega / k-\dot{z}_{0 i}} d p\right]$. 
Before proceeding with the solution, it proves convenient to change notation somewhat in order to simplify this equation. We will first redefine the $\dot{p}_{j}^{\top}$ and $\mathbf{s}_{j}$ so that all the $e_{1}$ become pogitlve. We make the change of variable defined by

$$
\dot{p}_{j}^{\tau^{\prime}}=\dot{p}_{j}^{\tau} \frac{e_{j}}{|e|}, \quad S_{j}^{\prime}=S_{j} \frac{e_{j}}{|e|} \text {. }
$$

We define also

$$
G=\pi a^{2} e^{2} g, \quad \dot{M}_{j}=\gamma_{j}^{3} m_{j}
$$

Using these definitions and $\mathrm{Eq}_{i},(80)$ we may rewrite $\mathrm{g} \nu_{\mathrm{i}}$ and obtain

$$
g \nu_{i}=g \frac{\pi a^{2} e^{2} n_{i}}{\gamma^{3} m_{i} c^{2}}=\frac{G_{i}}{M_{i} c^{2}}
$$

We define also

$$
\sigma \equiv 1+g \sum \nu_{i}=1+g \sum \frac{n_{i}}{M_{i} c^{2}}
$$

and substitute $v$ for $\dot{z}_{0}$. Equation (97) may now be written in the form

$$
\frac{\dot{p}_{j}^{\tau^{\prime}}}{G}=\sum_{i=1}^{N} \frac{v_{j}}{\sigma c} \int \frac{v_{i}}{c} \frac{\left(\dot{p}_{i}^{\prime} \frac{\partial f_{0 i}}{\partial p}+s_{i}^{\prime}\right)}{\omega / k-v_{i}} d p-\int \frac{\left(\dot{p}_{i}^{\tau^{\prime} \frac{\partial f}{\partial p}}+S_{i}^{\prime}\right)}{\omega / k-v_{i}} d p
$$

This gives a set of $N$ equations for the $N$ unknowns $\dot{p}_{j}{ }^{\prime \prime}$. Since $v_{j}$ is a function of $p$, this is in fact an integral equation for $\dot{p}_{j}^{\tau^{\prime}}$. Since by Eqs. (29) and (99)

$$
v_{j}=\bar{v}_{j}+p / M_{j}, \quad \bar{v} \equiv \bar{z},
$$

the simplest assumption for $\dot{p}_{j}^{\prime}$ that allows a solution of Eq. (102) is

$$
\dot{p}_{j}^{\tau^{\prime}}=\alpha_{j}+\beta_{j} p
$$


These arguments are exactly analogous to those used above in discussing Eq. (89). With the above assumption for $\dot{\mathrm{p}}_{j}^{\tau}, \mathrm{Eq}$. (102) may be written in the form

$$
F_{j}^{1}+F_{j}^{2} p=0
$$

Since this equation is valid for a range of values of $p$, in particular also for $p=0$, this yields the two equations.

$$
F_{j}^{1}=0 \text { and } F_{j}^{2}=0 \text {, }
$$

which are $2 \mathrm{~N}$ equations that suffice to find the $2 \mathrm{~N}$ quantities, $\alpha_{j}$ and $\beta_{j}$. Explicitly now, inserting Eqs. (103) and (104) into Eq. (102), we obtain for the part $F_{j}{ }_{j}=0$ the equation

$\frac{\alpha_{j}}{G}=\sum_{i=1}^{N} \int\left(\frac{\bar{v}_{j} v_{i}}{\sigma c}-1\right) \frac{\left(\alpha_{i}+\beta_{i} p\right) \frac{\partial f_{0 i}}{\partial p} d p}{\omega / k-v_{i}}+\sum_{i=1}^{N} \int\left(\frac{\bar{v}_{j} v_{i}}{\sigma c}-1\right) \frac{s_{i}}{\omega / k-v_{i}} d p$.

Similarly, the equation corresponding to $\mathrm{F}_{\mathrm{j}}{ }^{2}=0$ is

$\frac{\beta_{j}}{G}=\sum_{i=1}^{N} \int \frac{v_{i}}{M_{j} \sigma c^{2}} \frac{\left(\alpha_{i}+\beta_{i} p\right)^{\frac{\partial f}{\partial p}} \frac{d p}{\partial / k-v_{i}} d p}{\omega / \sum_{i}} \int \frac{v_{i}}{M_{j} \sigma c^{2}} \cdot \frac{S_{i} d p}{\omega / k-v_{i}}$

In Eq. (107); we have placed $\bar{v}_{j}$ under the integral sign, as it is independent of p.' We have also dropped the primes on the redefined $s_{i}^{\prime}$.

These two equations, or really $2 \mathrm{~N}$ equations, contain integrals of the

form

$$
\int \frac{\mathrm{p}^{\mathrm{n}} \frac{\partial \mathrm{f}_{0}}{\partial \mathrm{p}} \mathrm{dp}}{\omega / \mathrm{k}-\mathrm{v}}, \mathrm{n}=0,1,2,
$$

which may be simplified. We shall simplify these integrals, insert this result into Eqs. (107) and (108), and then solve these $2 \mathrm{~N}$ linear equations for the $2 \mathrm{~N}$ 
unknowns $\alpha_{j}$ and $\beta_{j}$. Consider first the integral of Eq. (109) with $n=1$. We have, uging Eq. (103) and the result $\int_{-\infty}^{\infty} \frac{\partial f_{0}}{\partial p} d p=\left.\left.\right|_{0} ^{\infty}\right|_{-\infty} ^{\infty} 0$,

$$
\begin{aligned}
\int_{-\infty}^{\infty} \frac{p \frac{\partial f_{0}}{\partial p} d p}{\omega / k-v} & =\int \frac{p \frac{\partial f_{0}}{\partial p} d p}{\omega / k-\bar{v}-p / M}+M \int \frac{\partial f_{0}}{\partial p} d p \\
& =\int \frac{[p+M(\omega / k-\bar{v})-p] \frac{\partial f_{0}}{\partial p} d p}{\omega / k-\nabla-p / \bar{M}} \\
& =\int \mathbb{M}\left(\frac{\omega}{k}-\bar{v}\right) \int \frac{\frac{\partial f_{0}}{\partial p} d p}{\omega / k-v}
\end{aligned}
$$

Next we will simplify Eq. (109), with $\mathrm{n}=2$. Using again the previous assumptionsiand the explicit p-dependence of $v$, we have

$$
\begin{aligned}
& \int_{-\infty}^{\infty} \frac{p^{2} \frac{\partial f_{0}}{\partial p} d p}{\omega / k-v}=\int \frac{p^{2} \frac{\partial f_{0}}{\partial p} d p}{\omega / k-\bar{v}-p / M}+M\left(\int p \frac{\partial f_{0} !}{\partial p} d p+n_{0}\right)
\end{aligned}
$$

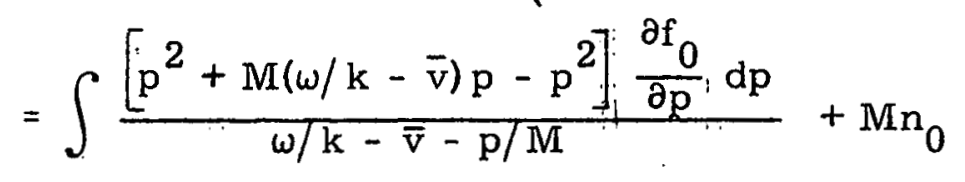

$$
\begin{aligned}
& =[M(\omega / k-\bar{v})]^{2} \int \frac{\frac{\partial f_{0}}{\partial p} d p}{\omega / k-v}+M n_{0} .
\end{aligned}
$$

To obtain thịs result we have used the relation

$$
-\int p \frac{\partial f_{0}}{\partial p} d p=n_{0}
$$

which is obtained by integrating by parts. In the last step of Eq. (111) we have also used Eq. $(110)$.

These results, Eqs. (110) and (111), will be used to simplify Eqs. (107) and (108). In particular the following term, common to Eqs. (107) and (108), may be written 


$$
\begin{aligned}
& \int \frac{v_{i}\left(\alpha_{i}+\beta p_{i}\right)}{\omega / k-v_{i}} \frac{\partial f_{0 i}}{\partial p} d p=\int\left(\bar{v}_{i} \alpha_{i}+\frac{p}{M_{i}} \alpha_{i}+\bar{v}_{i} p \beta_{i}+\frac{p^{2}}{M_{i}} \beta_{i}\right) \frac{\frac{\partial f_{0 i}}{\partial p / k-\bar{v}_{i}-p / M_{i}} d p}{\partial i} \\
& =\int\left[\bar{v}_{i} \alpha_{i}+\left(\omega / k-\bar{v}_{i}\right) \alpha_{i}+\bar{v}_{i} \beta_{i} M_{i}\left(\omega / k-\bar{v}_{i}\right)\right. \\
& +M_{i} \beta_{i}\left(\omega / k+\bar{v}_{i}\right) 2 \frac{\partial f}{\frac{\partial f}{\partial p} d p} \frac{\partial}{\omega / k-v_{i}}+\beta_{i} n_{0 i} \\
& =\int \frac{\omega}{k}\left[\alpha_{i}+\beta_{i} M_{i}\left(\omega / k-\bar{v}_{i}\right)\right] \frac{\frac{\partial f}{\partial p} d p}{\omega / k-v_{i}}+\beta_{i} n_{0 i} .
\end{aligned}
$$

To further simplify the notation, we adopt the definitions

$$
\int_{i} \equiv \int \frac{\frac{\partial f}{0 i} d p}{\omega / k-v_{i}}, \quad \int_{i}^{i} F(p) S_{i} \equiv \frac{F(p) S_{i} d p}{\omega / k-v_{i}}
$$

and will drop the subscript on $n_{0 i}$ and simply write $n_{i}$ : Using the above formulae, Eqs. (107) and (108) may be written in the form

$\frac{\alpha_{j}}{G}-\sum_{i=1}^{N}\left[\left(\frac{\bar{v}_{j}}{\sigma c} \frac{\omega}{k}-1\right)\left[\alpha_{i}+\beta_{i} M_{i}\left(\frac{\omega}{k}-\bar{v}_{i}\right)\right] \int_{i}+\frac{\bar{v}_{j}}{\sigma c} \beta_{i} n_{i}\right]=\sum_{i}^{N} \int_{i}\left(\frac{\bar{v}_{j} v_{i}}{\sigma c}-1\right) s_{i}$,

$\frac{\beta_{j}}{G}-\sum_{i=1}^{N}\left[\frac{\omega}{M_{i j} \sigma c^{2} k}\left[\alpha_{i}+\beta_{i} M_{i}\left(\frac{\omega}{k}-\bar{v}_{i}\right)\right] \int_{i}+\frac{\beta_{i} n_{i}}{M_{j} \sigma c}\right]=\sum_{i}^{N} \int_{i} \frac{v_{i}}{M_{j} \sigma c^{2}} S_{i}$.

The integrals in Eqs. (107) and (108) involving terms of the form of Eq. (109) with $\mathrm{n}=0,1,2$ have therefore been 'reduced to constant factors times the single integral with $\mathrm{n}=0$ only. This reduction was possible only because we assumed that the velocity spread in the beam was narrow enough so that the expansion, Eq. (29) or (104), was valid. 
In order to solve this pair of equations, (115) and (116), and thereby obtain the $\alpha_{j}$ and $\beta_{j}$ in terms' of the initial conditions, $s_{i}$, further manipulation of Eqs. (115) and (116) is necessary. The first step is to multiply Eq. (116) by $M_{j}\left(-\bar{v}_{j}+\frac{k \sigma c^{2}}{w}\right)$ and add it to Eq. (115), which then becomes, after canceling some terms,

$$
\frac{\alpha_{j}}{G}+\frac{\beta_{j} M_{j}}{G}\left(-\bar{v}_{j}+\frac{k \sigma c^{2}}{\omega}\right)-\sum_{i} \frac{k}{\omega} \beta_{i} n_{i}=\sum_{i}^{N} \int_{i}\left(\frac{k}{\omega} v_{i}-1\right) S_{i} .
$$

Next we multiply Eq. (116) by $\mathrm{M}_{j} \sigma \mathrm{c}^{2}$ to obtain

$\frac{\beta_{j} M_{j} \sigma c^{2}}{G}-\sum_{i}^{N}\left\{\frac{\omega}{k}\left[\alpha_{i}+\beta_{i} M_{i}\left(\frac{\omega}{k}-\bar{v}_{i}\right)\right] \int_{i}+\beta_{i} n_{i}\right\}=\sum_{i} \int_{i} v_{i} S_{i}$.

Equations (117) and (118), being a linear combination of Eqs. (115) and (116), are also soluble for the unknowns $\alpha_{j}$ and $\dot{\beta}_{j}$. We can obtain an equation involving only the $\beta_{j}$, by multiplying Eq: (117) by $\frac{\omega}{\mathrm{k}} \mathrm{G} \int_{j}$, summing over $\mathrm{j}$, and adding the result to, Eq. (118), which then gives

$$
\begin{aligned}
\beta_{j} M_{j}\left(\frac{\sigma c^{2}}{G}\right) & =\sum_{i}^{N} \beta_{i} M_{i}\left\{\left[\left(\frac{\omega}{k}\right)^{2}-\sigma c^{2}\right] \int_{i}+\frac{n_{i}}{M_{i}}\left[1+G \sum_{j} \int_{j}\right]\right\} \\
& =\sum_{i} \sum_{j}\left[\int_{i} v_{i} S_{i}\left(1+G \int_{j}\right)-\frac{\omega}{k} \int_{j} \int_{i} s_{i}\right] .
\end{aligned}
$$

To obtain this result we noted that $\sum_{i} F_{i}=\sum_{j} F_{j}$ and therefore cancelled two pairs of terms. This equation is of the form $\beta_{j} M_{j}=H$, where $H$ is independent of the index $j$. Since this equation is true for all the $N$ indices $j$, we have

$$
\beta_{j} M_{j}=\beta_{i} M_{i}, \quad \text { for } \quad i \neq j \text {. }
$$


With this result Eq. (119) may be rewritten

$$
\begin{aligned}
\beta_{j} M_{j}\left\{\frac{\sigma c^{2}}{G}-\left[\left(\frac{\omega}{k}\right)^{2}-\sigma c^{2}\right] \sum_{i} \int_{1}-\sum_{i} \frac{n_{i}}{V_{i}}\left(1+a \sum_{j} \int_{j}\right)\right\}_{j} \\
\quad=\sum_{i} \sum_{j}\left[\int_{i} v_{i} s_{i}\left(1+G \int_{j}\right)-\frac{\omega}{k} G \int_{j} \int_{i} s_{i}\right] .
\end{aligned}
$$

The identity Eq. (101) which defines $\sigma$ allows us to cancel two more pairs of terms, so that we obtain from Eq. (121)

$$
\beta_{j} M_{j}\left[\frac{c^{2}}{G}-\left(\frac{\omega}{k}\right)^{2} \sum_{i} \int_{i}+c^{2} \cdot \sum_{i} \int_{i}\right]=\sum_{i} \sum_{k}\left[\int_{i} v_{i} S_{i}\left(1+G \int_{k}\right)-\frac{\omega}{k} G \int_{k} \int_{i} S_{i}\right],
$$

or solving for $\beta_{j}$,

$$
\beta_{j}=\frac{\left[\sum_{i} \sum_{k} \int_{i} v_{i} S_{i}\left(1+G \int_{k}\right)-\frac{\omega}{k} \int_{i} S_{i} G \int_{k}\right]}{M_{j} c^{2}\left\{\frac{1}{G}+\left[1-\left(\frac{\omega}{k c}\right)^{2}\right] \sum_{i} \int_{i}\right\}_{i}}
$$

We have solved Eqs. (115) and (116) for $\beta_{j}$ (The shorthand integrals have been defined in Eq. (114).) The next step is to solve the equations for $\alpha_{j}$. This is almost trivial, as we need only insert the solution for $\beta_{j}$, into Eq. (117). First, however, we will simplify Eq. (117) using the result $\beta_{i} M_{i}=\beta_{j} M_{j}$. Substituting the value $\beta_{i}=\beta_{j} \mathbb{M}_{j} / M_{i}$ into $-\sum_{i} \frac{k}{\omega} \beta_{i} n_{i}$, we obtain, using also Eq. (101),

$$
-\sum_{i} \frac{k}{\omega} \beta_{i} n_{i}=-\beta_{j} M_{j} \frac{k}{\omega} \sum_{i} \frac{n_{i}}{M_{i}}=-\beta_{j} M_{j} \frac{k}{\omega}\left(\frac{\sigma c^{2}-c^{2}}{G}\right) \text {. }
$$


This result simplifies Eq. (117), which becomes, after canceling two terms,

$$
\alpha_{j}=\left(\bar{v}_{j}-\frac{k}{\omega} \mathrm{e}^{2}\right) \beta_{j} M_{j}+G \cdot \sum_{i} \int_{i}\left(\frac{k}{\omega} v_{1}-1\right) s_{i} .
$$

This gives the desired result for $\alpha_{j}$ almost immediately. Inserting Eq. (123) for $\beta_{j} M_{j}$ into this equation and canceling three pairs of terms gives

$$
\alpha_{j}=\frac{\sum_{i} \sum_{k}\left\{\int_{i} v_{i} S_{i}\left[\frac{\bar{v}_{j}}{c^{2}}\left(1+G \int_{k}\right)-G \frac{\omega}{k c^{2}} \int_{k}\right]-\int_{i} S_{i}\left[\left(\bar{v}_{j}-\frac{\omega}{k}\right) \frac{G}{c} \frac{\omega}{k} \int_{k}+1\right]\right\}}{\frac{1}{G}+\left[1-\left(\frac{\omega}{k c}\right)^{2}\right] \sum_{i} \int_{i}}
$$

We have now solved Eqs: (107) and (108) for $\alpha_{j}$ and $\beta_{j}$. Using Eq. (104), we. can also obtain $\dot{p}_{j}^{\tau^{\prime}}$. Since $\dot{p}_{j}^{\prime \tau^{\prime}}=\alpha_{j}+\beta_{j} p$ and, by Eq. (103), $v_{j}=\bar{v}_{j}+p / M_{j}$, it is clear that the equation for $\dot{p}_{j}^{\tau^{\prime}}$ is just Eq. (125), but with $\bar{v}_{j}^{\prime}$ replaced by $v_{i j}$. This new equation may be rewiritten in the form

$\dot{p}_{j}^{\tau^{\prime}}=\frac{\sum_{i} \sum_{j}\left(v_{j}-\frac{\omega}{k}\right) \frac{G}{c^{2}} \int_{k} \int_{i}\left(v_{i}-\frac{\omega}{k}\right) s_{i}+\frac{v_{j}}{c^{2}} \int_{i} v_{i} s_{i}-\int S_{i}}{\frac{1}{G}+\left[1-\left(\frac{\omega}{k c}\right)^{2}\right] \sum_{i} \int_{i}}$.

Inserting finally the full equations for the definitions adopted in Eq. (114), "we " obtain (inserting again the primes on $S_{i}^{\prime}$ )

$$
\dot{p}_{j}^{\tau^{\prime}}=\frac{\sum_{i=1}^{N} \sum_{k_{i}=1}^{N}\left[-\left(v_{j}-\frac{\omega}{k}\right) \frac{G}{c^{2}} \int \frac{\frac{\partial f}{0 k} d p}{\omega / k-v_{k}} \int S_{i}^{\prime} d p+\frac{v_{j}}{c^{2}} ! \int \frac{v_{i} S_{i}^{\prime}}{\omega / k-v_{i}} d p-\int \frac{1 \cdot S_{i}^{\prime}}{\omega / k-v_{i}} d p\right]}{\frac{1}{G}+\left[1-\left(\frac{\omega}{k c}\right)^{2}\right] \sum_{i=1} \int \frac{\frac{\partial f_{i}^{\prime}}{\partial p} d p}{\omega / k-v_{i}}} .
$$


We have now obtained the transform $\dot{\mathrm{p}}_{\mathrm{j}}^{\tau^{\prime}}$ in terms of the initial conditions, $S_{1}^{\prime}$, and in terms of the zero-order distribution functions. To go to the transforms $\dot{\mathrm{p}}_{j}^{T}$ and $\mathrm{S}_{i}$, which differ at most by -1 , we turn to Eqs. (98). The transform $S_{i}$, we recall, is defined in Eq. (86) as the spatial transform of the initial displacement. The problem is now in principle solved. If one desires the distribution function, one need only insert the result for $\dot{p}_{j}^{\tau}$ into Eq. (94) to obtain $\mathrm{f}_{i}^{\top}$. To obtain the potentials $\phi^{\top}$ and $\mathrm{A}^{\boldsymbol{T}}$ one inserts the result for $f_{i}^{\tau}$ into Eqs. (92) and (93). If one wants the actual space and time dependence of the fields, then one inserts the transforms into Eq. (36) and performs the integration. The fields $\underset{\sim}{\mathrm{E}}$ and $\underset{\sim}{\mathrm{B}}$ are now found from the usual relations

$$
\begin{aligned}
& \underset{\sim}{E}=-\frac{1}{c} \frac{\partial A}{\partial t}-\nabla \phi ; \\
& \underset{\sim}{B}=\nabla \times A .
\end{aligned}
$$

For completeness we note how Eq. (127) changes if we go to distribution function in velocity space. 'Suppose that the distribution function in velocity space is called $F_{0}(v)$. Then under the assumption that' it is 'also normalized to $\mathrm{n}_{0}$; we have'

$$
\int f_{0}(p) d p=n_{0}=\int_{i !} F_{0}(v) d v
$$

Also

$$
\int f_{0}(p) d p=\int f_{0}(p(v)) \frac{d p}{d v} d v
$$

Hence by Eq. (130) and also Eq. (103),

$$
f_{0}(p) \frac{d p}{d v}=f_{0} M=F_{0}
$$

Thus the integrals in Eq. (127)' become 


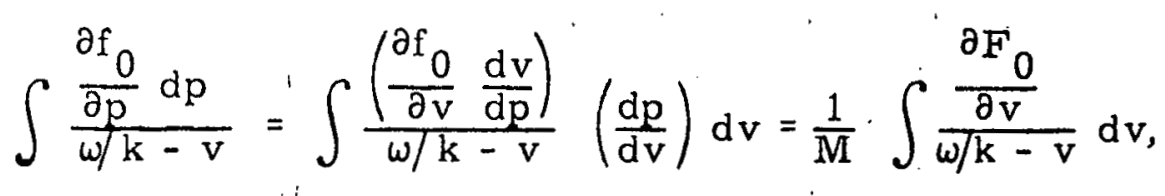

which indicates the transformation of these integrals when going to velocity space. To see how the $S_{i}$ integrals transform, we note that by the definition, Eq. (86), and using Eq. (132),

$$
S_{i}=f^{S \tau}(t=0)=\frac{1}{M} F_{0}^{S \tau}(t=0),
$$

where the superscript $s \tau$ indicates spatial transform. Thus

$$
\int S_{i} d p=\int S_{i} \frac{d p}{d v} d v=\int F_{0}^{s \tau}(t=0) d v
$$

where we have again used the result $d p / d v=M$. Thus the $S_{i}$ integrals are unchanged in form.

The solution, Eq. (127), that we have found for our problem is not at all convenient for practical calculation. 'In the next section we'shall show how' a useful dispersion relation may be obtained from it.

\section{THE DISPERSION RELATION}

In order to obtain a useful dispersion relation, we shall follow the Laplace transform method of Landau 1 and Jackson. ${ }^{2}$ The procedure is to invert the transform and thus to find the time dependence of the fields and the distribution function. The resulting integral is a contour integral, and if the integrand is analytically continued into the whole complex plane it may be evaluated by the residue theorem. The numerator of the integrand depends linearly on the initial conditions, and if these are sufficiently smooth the poles of the integrand will arise only from the zeros of the denominator. These zeros give the discrete frequencies of the system and hence constitute the dispersion relation. This denominator is independent of the initial conditions. 
In our case, because we have included retardation terms in evaluating the potentials, branch cuts appear, so that besides the previous discrete.'. frequencies one has branch cut integrals which, however, decay with time, Analysis indicates these to be associated with the finite signal velocity of light. These terms ensure that there is no signal at time $t$ further than ct from the beam. These terms therefore do not enter into ordinary experimental conditions. In the limit where the beams are infinitely wide the branch cut integrals disappear,' which explains why the integrals do not appear in the usual one-dimensional formulation of plasma problems.

\subsection{Analytic Continuation}

The first step in obtaining the dispersion relation is to invert the transform $\dot{\mathrm{p}}^{\tau}$, obtained in Eq. (127). (Recall that $\dot{\mathrm{p}}^{\tau}= \pm \dot{\mathrm{p}}^{\tau^{\prime}}$.) We wish to obtain the time dependence only and therefore follow the prescription given in Eq. (36): multiply by $e^{-i \omega t}$ and integrate over $\omega$ along the specified contour. However, the $\lambda_{j}$ of Eq. (36) are also functions of $\omega$, being solutions of Eq. (75). Hence we must first sum over $\lambda_{j}$ and then integrate over $\omega$. But the sum is uniformly convergent, so we may interchange the sum and integration signs, giving for the time and radial dependence of $\dot{p}_{z}^{\tau}$,

$$
\dot{p}_{z}^{k m}(r, t)=\frac{1}{2 \pi} \sum_{j=1}^{\infty} \int_{W} b_{j} J_{m}\left(\lambda_{j} r\right) e^{-i \omega t}\left(\dot{p}_{z}^{\tau}\right) d \omega .
$$

If the integrand has no branch cuts and vanishes over the large semicircle indicated in Fig. 2, then the integral along the line $W$ may be evaluated by the residue theorem. The function $\dot{p}_{z}^{\tau}$ defined in Eq. (127) is, however, defined only in the part of the $\omega$ plane above the line $W$. This follows from convergence and consistency requirements of the $t$ and $\omega$ integrations in. 
Eqs. (35) and (36). The line $W$ is above all singularities of the integrand of Eq. (36), or Eq. (127), and hence lies in the upper $\omega$ plane. That $\dot{p}_{z}^{\tau}$ is defined only above the line W is also eonglstent with the vandshing of the flrst term in Eq. (85) at $t=0$. We must therefore analytically continue the function defined in Eq. (127) into the lower $\omega$ plane.

The singularities of $\dot{\mathrm{p}}_{z}^{\tau}$ occur on the real $\omega$ axis. One is due to the quantity $G$ in Eq. (127), which, through Eqs. (99) and (80), is a function of $\lambda^{2}$, which is in turn, through Eq. (75), a function of $k \equiv\left(k^{2}-\omega^{2} / c^{2}\right)^{1 / 2}$. Since $\mathrm{K}_{\mathrm{m}}(\mathrm{ka})$ has branch points at $\omega= \pm \mathrm{kc}$ and $\omega= \pm \infty, \dot{\mathrm{p}}_{z}^{\tau}$ has these branch points. The contour indicated in Fig. 2 must therefore be modified to exclude these points. We shall defer further discussion of these singularities. The other factor of the integrand, $b_{j} J_{m}\left(\lambda_{j} r\right)$, is also a function of $\lambda_{j}$ and hence has these branch points too.

The other singularity of $\dot{\mathrm{p}}_{\mathrm{z}}^{\top}$ of Eq. (127) is a discontinuity along the real $\omega$ axis due to the term $\omega / \mathrm{k}-\mathrm{v}$ appearing in the denominator of various integrals. Let us consider these integrals in detail so that we may redefine them for $\omega$ in the lower half-plane in order that the new integrals will be continuous.

The discontinuous integrals are of the form

$$
I=\int_{-\infty}^{\infty} \frac{F(p)}{\omega / k-\bar{v}-p / M} d p
$$

Let us assume that the $F(p)$, which are functions of the zero-order distribution function or the initial perturbation, are analytic functions of $p$. We draw a contour in the p-plane as shown in Fig. 3. The integral of Eq. (137) may then be written, by the residue theorem, as

$$
\mathrm{I}_{+}=-\mathrm{II}_{+}+2 \pi \mathrm{i} \sum \operatorname{Res}\left(\mathrm{I}_{+}\right)
$$

where $I_{+}$is the integral along the real p-axis and $\mathrm{II}_{+}$is the integral along the semicircle with the integrand of Eq. (137). Note that $I_{+} \equiv I$ by Eq. (137). 
This equation is valid when the pole due to the zero of the denominator is assumed to be in the upper half of the p-plane, which will occur when $\omega$ is in the upper half of the $\omega$-plane, ( $k>0$ is assumed).

Suppose now that the point $\omega$ moves into the lower half $\omega$-plane, so that the pole at $p(\omega)$ also moves to the lower half $p$-plane. This is schematically indicated by Fig. 4. Equation (137) may again be rewritten, using the residue theorem, in the form

$$
I_{-}=-I_{-}+2 \pi i \sum \operatorname{Res}\left(I_{-}\right) \text {, }
$$

and again all quantities have the same definition as earlier, i. e., I_ is along the real p-axis and II _ is along the semicircle. Since one pole has left the closed contour of Fig. 3, there is one less term in the residue sum of Eq. (139). Consider now the case where in Fig. 3 the pole is infinitesimally above the real p-axis, and in Fig. 4 infinitesimally below the axis. Then evidently for the line integrals of Eqs. (138) and (139),

$$
\mathrm{II}_{+}=\mathrm{II}
$$

while

$$
\operatorname{Res}\left(I_{+}\right)-\operatorname{Res}\left(I_{-}\right)=\operatorname{Res}[\operatorname{pole} p(\omega)] \text {. }
$$

This means that the two integrals $\mathrm{I}_{+}$and $\mathrm{I}_{-}$differ by the residue at the pole $p=M(\omega / k-\bar{v})$ and are discontinuous on the real $\omega$-axis, as was to be shown. Explicitly the residue is

$$
\operatorname{Res}[\text { pole } p(\omega)]=F\left[p=M\left(\frac{\omega}{k}-\bar{v}\right)\right],
$$

where $F(p)$ is the numerator of Eq. (137). Thus

$$
I_{+}-I_{-}=2 \pi i \mathrm{~F}\left[\mathrm{p}=\mathrm{M}\left(\frac{\omega}{\mathrm{k}}-\overline{\mathrm{v}}\right)\right]
$$

gives the discontinuity as the pole crosses the real p-axis, or equivalently. as $\omega$ crosses the real $\omega$-axis. 
In order to analytically continue the function $\dot{\mathrm{p}}^{\tau}$ of Eq. (127) into the lower $\omega$-plane, we must redefine the integrals of that equation so that there is no discontinuity. This is now easily done. One changes the contour indicated in Fig. 3 so that when $\omega$ or $p(\omega)$ is in the lower plane, the contour still encloses the pole, as indicated in Fig. 4. With the new contour which defines I_',

$$
I_{-}^{\prime}=I_{-}+2 \pi i F\left[p=M\left(\frac{\omega}{k}-v\right)\right]
$$

when the pole is in the lower half-plane, and obviously when the pole is just below the p-axis,

$$
I_{+}=I_{-}^{\prime}
$$

by Eq. (143), so that the redefined integral is continuous.

It may be easily seen that when $k<0$, there is a minus sign in front of the $2 \pi \mathrm{i}$ of $\mathrm{Eq} .(143)$ and the contour is deformed so that when $\omega$ goes into the lower half $\omega$-plane, the pole $p(\omega)$ that enters the upper p-plane is excluded, as shown in Fig. 5 .

In summary, whether $k>0$ or $k<0$, when $\omega$ is in the upper $\omega$-plane the contour of integration for the integrals of Eq. (127) is along the real p-axis. When $\omega$ passes into the lower $\omega$-plane, the proper analytic continuation of Eq. (127) is obtained by deforming the path of integration for the integrals in the $\mathrm{p}$-plane. If $\mathrm{k}>0$, the path is deformed to that of Fig. 4, and if $\mathrm{k}<0$, the path is deformed to that of Fig. 5 .

We therefore have,

$$
\begin{aligned}
& I=\int_{C} \frac{F(p) d p}{\omega / k-\bar{v}-p / \bar{M}} \text { for } \operatorname{Im}(\omega)>0, \\
& I=\int_{C} \frac{F(p) d p}{\omega / k-\bar{v}-p / \bar{M}} \pm 2 \pi i F[p=M(\omega / k-\bar{v})] \text { for } \operatorname{Im}(\omega)<0,
\end{aligned}
$$


where $c$ is the real p-axis. When $\operatorname{Im}(\omega)=0$, the above two expressions will give the same limit. These are essentially the same arguments given by Landau $^{1}$ and Jackson. ${ }^{2}$

Let us now return to the other singularities of $\dot{\mathrm{p}}_{z}^{\tau}$, due to the terms $\lambda^{2}$. These occur in the quantity G of Eq. (127), defined by Eqs. (99) and (80). Through Eq. (75), $\lambda^{2}$ is a function of $\kappa=\left(k^{2}-\omega^{2} / c^{2}\right)^{1 / 2}$ and $K_{m}(\kappa a)$. Owing to the conditions on the convergence of the integral in Eq. (49) and the integration result, the previous functions are valid only for $\operatorname{Im}(\omega)>0$. (See) Eqs. (50) and (51) for the exact range of definition.) We must therefore examine the function $\mathrm{K}_{\mathrm{m}}(\kappa \mathrm{a}), \kappa^{2} \equiv \mathrm{k}^{2}-\omega^{2} / \mathrm{c}^{2}$, more carefully to determine the analytic continuation of $\lambda^{2}$ into the lower complex $\omega$-plane. We have 29

$$
K_{m}(z)=\sum_{r=0}^{m-1} c_{r} z^{2 r-m}+\sum_{r=0}^{\infty} d_{r} z^{m+2 r}\left(\log z-A_{r m}\right) .
$$

Hence the singularities of $K_{m}(k a)$ are those due to $\left(k^{2}-\omega^{2} / c^{2}\right)^{1 / 2}$ and $\log$ $\left(\mathrm{k}^{2}-\omega^{2} / \mathrm{c}^{2}\right)^{1 / 2}$. The singularities of the square root term are branch points at $\omega= \pm \mathrm{kc}$, while the singularities of the log term are branch points at $\omega= \pm \mathrm{kc}$ and at $\omega=\infty$. The function $\mathrm{K}_{\mathrm{m}}(\mathrm{ka})$ may therefore be made single-valued by branch cuts from $-\infty$ to $|k| c$, and from $|k| c$ to $\infty$, in the complexw-plane. To specify the functions in detail, consider $\log \left(\mathrm{k}^{2}-\omega^{2} / \mathrm{c}^{2}\right)^{1 / 2}=\cdot \frac{1}{2} \log$ $\left(k^{2}-\omega^{2} / c^{2}\right), k>0$, which may be written as

$$
\begin{aligned}
\log \left(k^{2}-\omega^{2} / c^{2}\right) & =\log [(\omega-k c)(\omega+k c)(-1)] \frac{1}{c^{2}} \\
& =\log \left|k^{2}-\omega^{2} / c^{2}\right|+i \arg (\omega-k c)+i \arg (\omega+k c)-i \pi \\
& =\log \left|k^{2}-\omega^{2} / c^{2}\right|+i \theta_{1}+i \theta_{2}-i \pi
\end{aligned}
$$


Thus

$$
\theta_{l} \equiv \arg \left(\mathrm{k}^{2}-\omega^{2} / \mathrm{c}^{2}\right)^{1 / 2} \equiv \arg \kappa=\frac{\theta_{1}+\theta_{2}-\pi}{2}
$$

It is easily verified, by reference to Fig. 6, that for $\omega$ in the upper complex plane, $-\pi / 2<\theta_{l}<\pi / 2$, which gives the same phase angle for $\kappa$ as obtained in Eq. (57). Thus the specification of $\theta_{l}$ in Eq. (149) is correct, and will therefore give the proper analytic continuation of $\kappa$ into the lower $\omega$-plane.

We still need the analytic continuation of $\lambda^{2}$. We therefore return to Eq. (75), which may be written in the form

$$
\lambda_{j} a \frac{J_{m+1}\left(\lambda_{j} a\right)}{J_{m}\left(\lambda_{j} a\right)}=\kappa a \cdot \frac{K_{m+1}(\kappa a)}{K_{m}(\kappa a)} \text {. }
$$

By writing out the explicit expansion of the $J_{m}$ Bessel functions, the lefthand side (L. H: S. ) of Eq.(150) may be written as

$$
\text { L.H. S. [Eq. (150)] }=\frac{\sum_{n=1}^{\infty} a_{n} \lambda^{2 n}}{\sum_{n=0}^{\infty} b_{n} \lambda^{2 n}}
$$

which is a function of $\lambda^{2}$. Hence, since the R. H. S. has been defined in the whole complex $\omega$-plane, $\lambda^{2}$ is obtained from Eq. (150), also for the whole. complex $\omega$-plane without any further analytic continuation. For the expansion. in Eq. (136) we need $\lambda_{j}$, while only $\lambda_{j}{ }^{2}$ is obtained from Eq. (150). This is fixed by choosing $\operatorname{Re}\left(\lambda_{j}\right)>0$, as in Eq. (39), to be valid for the whole complex plane.

We note too that division of Eq. (75) by $\mathrm{J}_{\mathrm{m}}\left(\lambda_{\mathrm{j}} \mathrm{a}\right) \cdot \mathrm{K}_{\mathrm{m}}(\mathrm{ka})$ to obtain Eq. (150) is valid because, first, $K_{m}(\kappa a)$ has no zeros in the range $-\pi / 2<\kappa a<\pi / 2$. (ref. $30)$, and secondly, because $J_{m}\left(\lambda_{j} a\right) \neq 0$ from the definition of the $\lambda_{j}$. 


\subsection{Branch Cuts}

Having now obtained the analytic continuation of the integrand of Eq. (136). we proceed to evaluate the integral by contour integration. The contour cannot, however, be that of Fig. 2, owing to the branch cuts indicated in Fig. 6. Hence the integrand is analytic only inside the contour indicated in Fig. 7. If the integral over the large semicircle tends to zero as its radius tends to $\infty$, application of the residue theorem to Eq. (136) gives

$$
\begin{aligned}
\dot{p}_{z}^{k m}\langle r, t\rangle=-i & \sum_{j=1}^{\infty}\left\{\sum_{n} e^{-i \omega_{n} t} \operatorname{Res}\left[b_{j} J_{m}\left(\lambda_{j} r\right) \dot{p}_{z}^{\tau}\right]_{\omega=\omega_{n}}\right\} \\
& -\sum_{j=1}^{\infty}\left[\int_{\infty}^{k c}(u)+\int_{k c}^{\infty}(l)+\int_{-k c}^{-\infty}(u)+\int_{-\infty}^{-k c}(l)\right] \frac{e^{-i \omega t}}{2 \pi}\left[b_{j} J_{m}\left(\lambda_{j} r\right) \dot{p}_{z}^{\tau}\right] d \omega .
\end{aligned}
$$

The sum over $\mathrm{n}$ is over the contributions from the $\mathrm{n}$ poles indicated in Fig: 7. The sign $\left(\begin{array}{l}u \\ i\end{array}\right)$ in the branch cut integrals indicates that the integral is over the (loper lower) part of the branch cut.

For some reasonable assumptions on the zero-order distribution function and the initial perturbation, we shall show that the integral on the semicircle tends to zero. Let us examine each term in Eq. (136) that depends on $w$. Consider first $\lambda$, obtained from Eq. (150). As $\omega \rightarrow \infty, \kappa \rightarrow \infty$, and the $\mathrm{K}_{\mathrm{m}}$ ( $(\kappa a)$ Bessel functions may be replaced by their asymptotic form, which is the same for all m. Hence the R.H.S. of Eq. (150) equals $\kappa a$. This is a large number and clearly then; as $\kappa \mathrm{a} \rightarrow \infty$, the solutions of Eq. (150) are near the roots of $J_{m}\left(\lambda_{j} a\right)$. These roots are small finite numbers for small j. Hence the quantity $G$ in Eq. (127), defined through Eqs. (99) and (80), behaves like

$$
\frac{1}{\mathrm{G}} \sim \omega^{2} \text {. }
$$


Consider next the coefficient $b_{j}$ in Eq. (136), defined in Eq. (37). Its dependence on $\omega$ is given by its dependence on $h$, defined in Eq. (73). Since for large $k$ a the derlvatives of $\mathrm{K}_{\mathrm{m}}(k \mathrm{k})$ aro proportongl to $\mathrm{K}_{\mathrm{m}}(\mathrm{k})$, th clear that $h \sim k a$, and hence (for small $j$ )

$$
b_{j} \sim 1 / \omega^{2}
$$

At certain points in the $\omega$-plane, the denominator of Eq. (37) may blow up (where $h^{2}+\lambda_{j}^{2}-m^{2} / a^{2}=0$ )." This is, however, a spurious effect. Examination of Eqs.(35) and (36) shows that the term $f_{1}^{\tau}$ (or $\phi^{\tau}$, etc.) must go to zero if the denominator of $b_{j}$ goes to zero, because $f_{1}(r)$ (or $\phi(r)$, etc.) must clearly be finite. The transition to the limit where the denominator of $b_{j}$ is zero is smooth and is given by Eq. (40), where it may be seen explicitly that $b_{j}$ contributes no poles. The cancellation of the denominator of $b_{j}$ as it approaches zero comes from the term $S^{\prime}$ in $\dot{p}^{\tau}$ of Eq. (127), which is the spatial transform of the initial perturbation.

When $\mathrm{h}^{2}+\lambda_{\mathrm{j}}^{2}-\mathrm{m}^{2} / \mathrm{a}^{2}=0$, then for large $\omega, \lambda_{\mathrm{j}} \sim \mathrm{h} \sim \omega$ and thus $\lambda_{\mathrm{j}}$ is large, and it appears from Eq. (40) that $b_{j} \sim \lambda_{j}^{2} \sim \omega^{2}$. However, here too we must now include the term $\mathbf{S}^{\prime}$, the spatial transform, and use certain theorems in Fourier-Bessel theory which show that $b_{j} S^{\prime} \sim 1 / \sqrt{\lambda}$ for large $\lambda$, so that this factor does decrease with $\lambda$.

Consider next the various integrals of the form $F(p) /(\omega / k-v)$ appearing in $\dot{p}^{\tau}$, Eq. (127). Since we have assumed a linear expansion of $v$ in powers of $\mathrm{p}$ (see Eq. (29)), the range of $\mathrm{p}$ was assumed to be narrow in the various distribution functions. Therefore we can write, for large $\omega$,

$$
\int \frac{F(p) d p}{\omega / k-v}=\frac{k}{\omega} \int F(p)\left[1+v \frac{k}{\omega}+\left(\frac{v k}{\omega}\right)^{2}+\ldots\right] d p .
$$


When $F(p)=\partial f_{0} / \partial p$, the first term vanishes. Since $v=\bar{v}+p / M$, the nonvanishing part of the second term is proportional to $\int \frac{\partial f_{0}}{\partial p} p d p=-n_{0^{*}}$. Since this is unequal to zero, we have then

$$
\int \frac{\partial f / \partial p}{\omega / k-v} d p \sim \frac{1}{\omega^{2}}
$$

Consider now the other possible form for $F(p)$, viz. $F(p)=S$. If $S$ is odd in p, then the first term in Eq. (155) is zero; but if $S$ is even in $p$, then the first term does not vanish. Hence we have

$$
\frac{S}{\omega / \mathrm{k}-\mathrm{v}} \sim \frac{1}{\omega} \text { or } \frac{1}{\omega^{2}}
$$

From the above results we see that the numerator of $\dot{p}^{\top}$ in Eq. (127) goes at least like $1 / \omega$, while the denominator goes like $\omega^{2}$. Hence

$$
\dot{\mathrm{p}}^{\tau} \sim 1 / \omega^{3}
$$

Returning to Eq. (136) and noting also from Eq. (154) that $b_{j} \sim 1 / \omega^{2}$, we see that the line integral does indeed vanish on the large semicircle of Fig. 7. Hence the result indicated in Eq. (152) is correct.

Let us now look for the poles at $\omega=\omega_{n}$ in Eq. (152). As pointed out above, $b_{j}$ cannot contribute poles, Hence the poles can arise only from $\dot{p}^{\tau}$, defined by Eqs. (98) and (127). On the assumption that the initial perturbations in the beams are sufficiently smooth, the numerator of Eq. (127) will not contribute any poles. Thus poles can arise only from the zeros of the denominator. Hence the poles $\omega_{\mathrm{n}}$ arise from the solutions of

$$
-\frac{\lambda^{2}+\kappa^{2}}{4 \pi e^{2}}=\left[1-\left(\frac{\omega}{k c}\right)^{2}\right] \sum_{i=1}^{N} \int \frac{\partial f_{0 i} / \partial p}{\omega / k-v_{i}} d p
$$


where we have used Eqs. (80) and (99) to rewrite G. These integrals may be written in terms of the velocity by use of Eq. (133). We recall again the definitions from Eqs. (29) and (57),

$$
v_{i}=\bar{v}+\frac{1}{\lambda^{3} m_{i}} p, \quad k=\left(k^{2}-\omega^{2} / c^{2}\right)^{1 / 2}
$$

The $\lambda^{\prime}$ s are functions of $k$ and $\omega$ through Eq. (150),

$$
\lambda_{j} a \frac{J_{m+1}\left(\lambda_{j}^{a}\right)}{J_{m}\left(\lambda_{j} a\right)}=\kappa a \frac{K_{m+1}(k a)}{K_{m}(\kappa a)} \text {. }
$$

Equation (159) is valid throughout the complex $\omega$-plane. The analytic continuation of the p-integrals is given by Eq. (146), while the argument of $k$ is given by Eq. (149).

Equations (159)-(161) are the desired dispersion relations. They give the discrete frequencies of the system, and show whether the system is stable or unstable and whether it emits radiation or not. These give the main new result of this paper.

We turn now to the physical significance of the branch cut integrals in Eq. (152). Examination of this result shows it to be similar to a situation discussed by Brillouin ${ }^{10}$ (and summarized by Stratton ${ }^{11}$ ). They consider a harmonic oscillation at $z=0$ and $t=0$ at the surface of a semi-infinite dispersive medium and inquire into the propagation of the signal at later time into the medium. The result is that the wave at any point in the medium may be represented by a single frequency and two branch cut integrals, in a manner similar to Eq. (152). These branch cut integrals decay with time, but establish casuality, i.e., that no point in the medium can oscillate at a time earlier than $t^{\prime}=z / c$, where $z$ is the distance to the source oscillation. These branch cut 
integrals therefore cancel that part of the single frequency term at a distance greater than $z^{\prime}=c t^{\prime}$.

We shall sketch briefly some of the analogous arguments in Eq. (152). Let us consider first the discrete frequency terms. We have assumed that the numerator of $\dot{\mathrm{p}}^{\tau}$. in Eq. (127) has no poles in the $\omega$-plane, which required that the initial perturbation be a sufficiently smooth function of $p$. Nevertheless, the spatial dependence of the initial perturbation may be chosen so that $S$, defined in Eq. (86), has the factors $\delta_{\lambda \lambda_{0}}{ }^{\delta} \mathrm{mm}_{0} \delta\left(\mathrm{k}-\mathrm{k}_{0}\right)$. Then by Eq. (127), $\dot{\mathrm{p}}^{\tau}$ has these $\delta$-functions as a factor too, and from the discrete frequency terms of Eqs. (152) and (36),

$\dot{p}(r, \theta, z, t)=e^{i m_{0} \theta+i k_{0} z} \sum_{n}^{N} A_{n} e^{-i \omega_{n} t} J_{m_{0}}\left(\lambda_{0} r\right) \quad$ for $\quad\left\{\begin{array}{l}r \leq a, \\ t>0,\end{array}\right.$

In a similar manner it is possible to find the value of the fields outside the cylinder also. From Eqs. (70), (72), (93), and (94), we see that when $\dot{\mathrm{p}}^{\tau}$ has these $\delta$-functions, we have

$$
\phi(r, \theta, z, t)=e^{i m_{0} \theta+i k_{0} z}: \sum_{n}^{N} B_{n} e^{-i \omega_{n} t} K_{m_{0}}\left(\lambda_{0} r\right) \text { for }\left\{\begin{array}{l}
r \geq a, \\
t>0,
\end{array}\right.
$$

with a similar expression for A. It is clear from this equation that at any time a disturbance may be found at an arbitrary distance from the cylinder. The effect of including the branch cut terms must be, clearly, to maintain causality, so that there is no signal at sufficiently distant spatial points. There will also be branch cut terms in the expression for $\phi$ within the cylinder. The physical significance of these terms is less obvious. 
Finally, we will show that the branch cut integrals decay with time, and in fact decay as $1 / t^{n}$, where $n$ is as large as one wishes, if $t$ is large enough. Let us write a typical integral of Eq. (152) in the form.

$$
B=\int_{k c}^{\infty} e^{-i \omega t} F(\omega) d \omega
$$

For $t>>k c$, the exponential term oscillates very rapidly with $\omega$, while the term $F(\omega)$ is a comparatively smooth function of $\omega$. Hence the integral decays with time.

Next we show that for $t$ large enough the branch cut integrals decay as $1 / t^{n}$. We may rewrite the integral in the following manner, after integrating by parts:

$$
\int_{k c}^{\infty} e^{-i \omega t} F(\omega) d \omega=\left.\frac{e^{-i \omega t} F(\omega)}{-i t}\right|_{k c} ^{\infty}+\frac{1}{i t} \int e^{-i \omega t} \frac{d}{d \omega}[F(\omega)] d \omega .
$$

The first term evaluated at the upper limit gives zero, because we have shown above that $F(\omega) \sim 1 / \omega^{5}$ for large $\omega$. Before considering the lower limit, we shall group the two branch cut terms due to, say, the right-hand branch cut of Fig. 7. We then have,

$$
B_{R}=\frac{e^{-i \omega t}}{-i t}\left[F(\omega)_{u}-F(\omega)_{l}\right]_{\omega=k c}+\frac{1}{i t} \int_{k c}^{\infty} e^{-i \omega t}\left[F^{\prime}(\omega)_{u}-F^{\prime}(\omega)\right] d \omega
$$

where $u$ and $l$ indicate the upper and lower sides of the cut. The only quantity in $F(\omega)$ which differs on the two sides of the cut is $\lambda^{2}$. This may be determined from Eq. (150), which may be rewritten in the following form by use of Eqs. (147) and (151):

$$
\frac{\sum a_{n} \lambda^{2 n}}{\sum b_{n} \lambda^{2 n}}=\frac{\sum_{n=0}^{m} c_{n} z^{2 n}+z^{2 m} \sum_{n=1}^{\infty} d_{n} z^{2 n}\left(\log z^{2}-A_{m}\right)}{\sum_{n=0}^{m=1} f_{n} z^{2 n}+z^{2 m} \sum_{n=0}^{\infty} g_{n} z^{2 n}\left(\log z^{2}-A_{m-1}\right)} .
$$


Here $Z \equiv \kappa a$. If $\lambda$ is desired, it is obtained from $\lambda^{2}$ uniquely, with the proviso $\operatorname{Re}(\lambda)>0$. Above the cut, $\arg (Z)=-\pi / 2$, while below $\arg (Z)=+\pi / 2$, as is elear.from Fq. (149). We are interested in the limlt of the above equation when $Z \rightarrow 0$. Different values of the R. H. S. will occur only in the log term. However; as $Z \rightarrow 0$, the imaginary part of the log function becomes negligible relative to the real part, so that the R. H.S. has the same value in this limit above and below the cut. Hence at $\omega=k c, \lambda^{2}$ is continuous, so that the first two terms in Eq. (166) equal zera.

The remaining term in Eq. (166) may again be integrated by parts. Again the value of $F^{\prime}(\omega)$ at $\omega=\infty$ is zero, because $F(\omega) \sim 1 / \omega^{5}$. Clearly all derivatives will be zero at $\omega=\infty$. Equation (166) may then be written as

$B_{R}=-\frac{e^{-i \omega t}}{(i t)^{2}}\left[F^{\prime}(\omega)_{u}-F^{\prime}(\omega)_{l}\right]_{\omega=k c}+\frac{1}{(i t)^{2}} \cdot \int_{k c}^{\infty} e^{-i \omega t}\left[F^{\prime \prime}(\omega)_{u}-F^{\prime \prime}(\omega)_{l}\right] d \omega$ $F^{\prime}(\omega)$ now contains also terms like $d \lambda^{2} / d \omega$, besides $\lambda^{2}$. However, we may write

$$
\frac{d \lambda^{2}}{d \omega}=\frac{d \lambda^{2}}{d z^{2}} \cdot \frac{d z^{2}}{d \omega}+\frac{d \lambda^{2}}{d z^{2}}\left(\frac{-2 \omega}{c^{2}}\right)
$$

Since inspection of Eq. (167) shows that $d \lambda^{2} / d Z^{2}$ at $Z=0$ has the same value above and below the cut, $d \lambda^{2} / d \omega$ is also continuous at $z=0$. Hence the first pair of terms in Eq. (168) are again zero. Similarly $d^{\nu} \lambda^{2} / d \omega^{\nu}$ for any integer $\nu$ is continuous at $\omega=\mathrm{kc}$, above and below the cut, so that repeated integration by parts of Eq. (168) will give zero for the first pair of terms. Hence, in. general, for any integer $\nu$

$$
B_{R}=\frac{1}{(i t)^{\nu}} \int_{-\infty}^{\infty} e^{-i \omega t}\left[F^{\nu}(\omega)_{u}-F^{\nu}(\omega)_{l}\right] d \omega
$$


or

$$
\mathrm{B}_{\mathrm{R}} \leq \frac{1}{\mathrm{t}^{\nu}} \int_{\mathrm{kc}}^{\infty}\left|\mathrm{F}^{\nu}(\omega)_{\mathrm{u}}-\mathrm{F}^{\nu}(\omega)_{l}\right| \mathrm{d} \omega \equiv \mathrm{B}_{\mathrm{R}}^{\nu}
$$

Hence if $B_{R}^{\nu+1}<B^{\nu}$, the time behavior of $B_{R}$ will be

$$
B_{R} \sim \frac{1}{t^{\nu+1}}
$$

This will occur for times so large that

$$
t>\frac{\int_{\mathrm{kc}}^{\infty}\left|\mathrm{F}^{\nu+1}(\omega)_{\mathrm{u}}-\mathrm{F}^{\nu+1}(\omega)_{l}\right| \mathrm{d} \omega}{\int_{\mathrm{kc}}^{\infty}\left|\mathrm{F}^{\nu}(\omega)_{\mathrm{u}}-\mathrm{F}^{\nu}(\omega)_{l}\right| \mathrm{d} \omega} .
$$

Hence, as was to be shown; $B \sim 1 / t^{n}$ for large enough $t$.

The branch cut terms we have obtained will not affect the usual Landau damping, because it occurs only for $\omega / k<c$, while the branch cut integrals occur only for $\omega / \mathrm{k}>\mathrm{c}$.

These branch cut integrals contain no poles, because this would imply that the denominator is zero, i. e., that the dispersion relations, Eqs. (159)(161) have a root, $\omega$ real, for $\omega / k c>1$. Since the $K_{m}$ are complex if $\omega / k c>1$, this is impossible.

These branch cut terms vanish in the limit of large a, i. e., large cylinder radii, and hence do not appear in the uaual one-dimensional formulation of plasma oscillations. This is easily seen. As a $\rightarrow \infty$, the $K_{m}$ Bessel functions approach the same asymptotic limit, so that the R. H.S. of Eq. (150) approaches $\mathrm{ka}$. Hence the solutions of Eq. (150) are near the zeros of $\mathrm{J}_{\mathrm{m}}(\lambda a)$, i. e., $\lambda \mathrm{a} \approx$ small numbers, and hence $\lambda$ vanishes as $1 / \mathrm{a}$. Since the branch cut integrals give a nonzero contribution only because $\lambda$ differs on the two sides of the cut, we see that as $\lambda \rightarrow 0$ the branch cut integrals approach zero. 
We have now solved our problem. The time behavior of the system is given by Eq. (152), where the frequencies $\omega_{n}$ are obtained from the dispersion. relations, Eqs. (159)-(161). For decaying solutions where $\omega / k<c, 1$. e. ' Landau damping, the branch cut terms certainly do not contribute. However, if $\omega / k>c$, the branch cut terms may be important. For the growing solutions, for all $w / k$, the decaying terms will quickly become negligible.

\section{COMPARISON WITH PREVIOUS WORK}

The dispersion relation obtained in Eqs. (159)-(161) is more general than earlier derivations. In this section we shall obtain the results of a number of other authors and point out the appropriate range of applicability of their results.

We shall first derive the result of Bludman et al., 4 who consider a hot relativistic electron stream penetrating a cold plasma. The radius of the cylinder is assumed infinite. Next we will obtain the results of Budker, ${ }^{5}$ who considers a cold relativistic electron beam traversing a stationary cold ion beam. Here the cylinder radii are finite. From these results we obtain next the results of Dawson and Oberman, ${ }^{3}$ who consider cold, stationary "beams" of finite radii; and also those of Sturrock, ${ }^{6}$ and Finkelstein and Sturrock ${ }^{14}$ who consider very thin relativistic beams.

Finally we comment on the results of Trivelpiece and Gould, ${ }^{31}$ and Granatstein, Schlesinger, and Vigants, ${ }^{32}$ who consider a cold stationary plasma cylinder, and show how these results, too, fit within the framework of our dispersion relation. 


\subsection{Infinitely Wide Streams}

The situation considered by Bludman et al. is that of a hot relativistic electron beam passing through a cold stationary plasma. To obtain their dispersion relation for the longitudinal oscillations of the system, from Eq. (159), we need first to derive an expression for the integral term in Eq. (159), when $f_{0}$ is a $\delta$ function. We will do this by letting $f_{0}$ be a square pulse function and then letting its width go to zero. We therefore define

$$
f_{0}=\left\{\begin{array}{l}
n_{0} / \Delta \text { for }-\Delta / 2 \leq p \leq \Delta / 2, \\
0 \text { otherwise. }
\end{array}\right.
$$

As mentioned in Eq. (11), $f_{0}$ is normalized to $\mathrm{n}_{0}$, the particle density. The function defined by Eq. (174) is not analytic in p. However, by smoothing the corners we may obtain an analytic function which is close to the above definition. This limiting process may be easily applied to what follows, so that as a shorthand we will use the above definition.

A typical integral term in Eq. (159) may then be written as

$$
\begin{aligned}
\mathrm{T}_{1} \equiv \int \frac{\frac{\partial \mathrm{f}_{0}}{\partial \mathrm{p}} \mathrm{dp}}{\omega / \mathrm{k}-\overline{\mathrm{v}}-\mathrm{p} / \gamma^{3} \mathrm{~m}_{0}} & =\frac{\mathrm{n}_{0}}{\Delta} \int \frac{\left[\delta\left(\mathrm{p}+\frac{\Delta}{2}\right)-\delta\left(\mathrm{p}-\frac{\Delta}{2}\right)\right]}{\omega / \mathrm{k}-\overline{\mathrm{v}}-\mathrm{p} / \gamma^{3} \mathrm{~m}_{0}}= \\
& =-\frac{\mathrm{n}_{0}}{\Delta}\left[\frac{1}{\omega / \mathrm{k}-\overline{\mathrm{v}}-\Delta / 2 \gamma^{3} \mathrm{~m}_{0}}-\frac{1}{\omega / \mathrm{k}-\overline{\mathrm{v}}+\Delta / 2 \gamma^{3} \mathrm{~m}_{0}}\right] \\
& =-\frac{\mathrm{n}_{0}}{\gamma^{3} \mathrm{~m}_{0}} \frac{1}{(\omega / \mathrm{k}-\overline{\mathrm{v}})^{2}-\left(\Delta / 2 \gamma^{3} \mathrm{~m}_{0}\right)^{2}} .
\end{aligned}
$$

Finally, letting $\Delta \rightarrow 0$, we obtain the result

$$
\operatorname{Lim}_{\Delta \rightarrow 0} \int \frac{\frac{\partial f_{0}}{\partial p} d p}{\omega / k-v}=\frac{-n_{0}}{\gamma^{3} m_{0}} \frac{1}{(\omega / k-\bar{v})^{2}}
$$

for the value of the integral where the particle species are cold. 
We may now write Eq. (159) explicitly for the case where there are three particle species. One of these species is the relativistic beam for which the term is left unchanged. Its distribution function will be denoted by $f_{-}$. The other two particle species are the cold background plasma, for which we will use Eq. (176). Since the particle ion and electron density are assumed roughly equal, say $n_{p}$, and the plasma is stationary, Eq. (176) gives for these terms

$$
T_{\text {plasma }}=\frac{-n p^{2}}{\omega^{2}}\left(\frac{1}{m_{+}}+\frac{1}{m-}\right) \approx \frac{-n p}{m-\frac{k^{2}}{\omega^{2}}} .
$$

Thus Eq. (159) becomes

$$
\frac{\lambda^{2}+k^{2}}{\left[1-(\omega / k c)^{2}\right]}=\frac{4 \pi e^{2} n_{p}}{m-} \frac{k^{2}}{\omega^{2}}+\int \frac{\frac{\partial f}{\partial f} d p}{v_{-}-\omega / k} .
$$

The $\lambda^{\prime}$ s are to be obtained from Eq. (161). When an infinitely wide beam is considered, $a \rightarrow \infty$ and the $K_{m}$ Bessel functions approach the same limit independently of $\mathrm{m}$. Hence R.H.S. of Eq. $(161) \rightarrow \infty$, and the solutions of the equation approach the zeros of $J_{m}\left(\lambda_{j} a\right)$. Since these are small numbers, for small $j$ and small $m$ (e.g., for $\left.m=0, \lambda_{1} a=2.4\right)$, clearly $\lambda_{j} \rightarrow 0$. Hence recalling the definition, Eq. (57), $\kappa^{2}=k^{2}-\omega^{2} / c^{2}$, we may write

$$
\frac{\lambda^{2}+k^{2}}{1-(\omega / k c)^{2}} \approx \frac{k^{2}-\omega^{2} / c^{2}}{1-(\omega / k c)^{2}}=k^{2} .
$$

Let us turn next to the second term on the R. H. S. of Eq. (178). It may be written in terms of distribution functions normalized to $\mathrm{n}_{\text {- }}$ in velocity space using Eq. (133). Together with Eq. (179) we may now rewrite Eq. (178) as 


$$
k^{2}=\frac{4 \pi e^{2} n_{p}}{m_{-}} \frac{k^{2}}{\omega^{2}}+\frac{4 \pi e^{2}}{\gamma{ }^{3} m} \int \frac{\frac{\partial F_{-}}{\partial v} d v}{v_{-}-\omega / k}
$$

With the usual definition

$$
\omega_{p}^{2} \equiv \frac{4 \pi e^{2} n_{p}}{m_{-}}
$$

we may rewrite Eq. (180) as

$$
\omega^{2}=\omega_{p}^{2}+I_{F}
$$

where

$$
I_{F} \equiv \frac{4 \pi e^{2} \omega^{2}}{\gamma^{3} m_{-} k} \int \frac{\frac{\partial F}{\partial v} d v}{v k-\omega} .
$$

The dispersion relation of Bludman et al. ${ }^{33}$ is just Eq. (182), but instead of $I_{F}$, their term is $I_{z z}{ }^{34}$ defined as

$$
I_{z z}=4 \pi e^{2} \omega \frac{f_{0 B}\left(v_{0}+\nu_{z}\right)}{\Omega^{1}-\nu_{z} k_{z}} \frac{\nu_{z}}{\theta_{\|}} \mathrm{d}^{3} \nu .
$$

We must therefore show that

$$
I_{F}=I_{z Z}
$$

in order to show that the dispersion relations are the same. They define $\mathrm{v}=\mathrm{v}_{0}+\nu_{\mathrm{z}}, \Omega^{\prime}=\omega-\mathrm{k}_{\mathrm{z}} \mathrm{v}_{0}$, and use $\nabla \mathrm{p}_{\mathrm{z}} \mathrm{f}_{0 \mathrm{~B}}=\frac{-\nu \|}{\theta} \mathrm{f}_{0 \mathrm{~B}}$. With these substitutions and integrating over $d v_{x} d v_{y}$ (since $d^{3} \nu=d v_{z} d v_{x} d v_{y}$ ), Eq. (184) becomes

$$
I_{z z}=4 \pi e^{2} \omega \int \frac{\frac{\partial f_{0 B}}{\partial p_{l}} v d v}{v k-\omega} .
$$

We have written $p_{l}$ for the linear momentum, $m \dot{z}$. To convert $p_{l}$. to the quantity $p$ used in our calculations, we use Eqs. (27), (13), and (20) to write 


$$
\mathrm{p}=\mathrm{p}_{\mathrm{z}}-\overline{\mathrm{p}}_{\mathrm{z}}=\mathrm{m} \dot{\mathrm{z}}+\frac{\mathrm{e}}{\mathrm{c}} \mathrm{A}_{\mathrm{z}}-\overline{\mathrm{p}}_{\mathrm{z}} \approx \mathrm{mz}+\frac{\mathrm{e}}{\mathrm{c}} \mathrm{A}_{0}-\overline{\mathrm{p}}_{\mathrm{z}}=\mathrm{mz}-\overline{\mathrm{m}} \overline{\mathrm{z}}
$$

This result is correct to lowest order in $\mathrm{A}_{0^{\circ}}$ Since $\overline{\mathrm{m}} \overline{\mathrm{z}}$ is a constant,

$$
\mathrm{dp}=\mathrm{d}(\mathrm{m} \dot{z}) \equiv \mathrm{dp} \cdot l
$$

We may also write $\mathrm{v}$ in Eq. (186) as $\mathrm{v}$ defined in Eq. (29), because Eq. (186) is only valid for a small range of velocities also. Hence

$$
\frac{\partial \mathrm{f}_{0 \mathrm{~B}}}{\partial \mathrm{p}_{l}}=\frac{\partial \mathrm{f}_{0 \mathrm{~B}}}{\partial \mathrm{p}}=\frac{\partial \mathrm{f}_{0 \mathrm{~B}}}{\partial \mathrm{v}} \frac{\partial \mathrm{v}}{\partial \mathrm{p}}=\frac{\partial \mathrm{f}_{0 \mathrm{~B}}}{\partial \mathrm{v}} \cdot \frac{1}{\gamma^{3} \mathrm{~m}} \text {. }
$$

Since $f_{0 B}$ is now a function of $v$, let us redefine it as $F_{0}$. Equation (186) now becomes

$$
I_{z z}=\frac{4 \pi e^{2} \omega}{\gamma^{3} m} \int \frac{\frac{\partial F_{0}}{\partial v} v d v}{v k-\omega} .
$$

Also, $\int \frac{\partial F_{0}}{\partial v} d v=0$, so that we have

$$
\int \frac{\frac{\partial F_{0}}{\partial v} v d v}{v k-\omega}=\int \frac{\frac{\partial F_{0}}{\partial v} v d v}{v k-\omega}-\frac{1}{k} \int\left(\frac{v k-\omega}{v k-\omega}\right) \frac{\partial F_{0}}{\partial v} d v=\frac{\omega}{k} \int \frac{\frac{\partial F_{0}}{\partial v} d v}{v k-\omega} .
$$

With this last result, Eq. (190) becomes

$$
I_{z z}=\frac{4 \pi e^{2}}{\gamma 3 m_{-}} \frac{\omega^{2}}{k} \int \frac{\frac{\partial F_{0}}{\partial v} d v}{v k-\omega}
$$

which by inspection is seen to be the same as $I_{F}$ of Eq. (183). We have therefore shown that $I_{F}=I_{z z}$ and hence that the Bludman et al. equation is the same as ours.

We note, however, that although Eq. (182) is the dispersion relation of Bludman et al. for the case of infinite radius, irrespective of $\omega / k$, our derivation of this equation assumed that $k a \gg 1$, i. e., $\left(k^{2}-\omega^{2} / c^{2}\right)^{1 / 2} a>1$. 
This inequality evidently breaks down when $\omega \approx k c$, so that the Bludman et al. equation is not valid for this domain, and one must return to our more accurate dispersion relation.

\subsection{Cold Beams and Budker's Results}

We turn now to Budker's results. He considers a cold relativistic electron beam and a cold stationary ion beam. Since there are two beams we need only two integral terms of Eq. (159). These may be obtained from Eq. (176). Denoting the ion term by $\mathrm{T}_{+}$and the electron term by $\mathrm{T}_{-}$, we obtain

$$
\mathrm{T}_{+}=\frac{-\mathrm{n}_{+} \mathrm{k}^{2}}{\mathrm{~m}_{+} \omega^{2}}, \quad \mathrm{~T}_{-}=\frac{-\mathrm{n}_{-}}{\gamma^{3} \mathrm{~m}_{-}\left(\omega / \mathrm{k}-\mathrm{v}_{-}\right)^{2}}
$$

with obvious notation. Collecting terms and inserting into Eq. (159) we obtain

$$
\frac{\mathrm{n}_{+} \mathrm{k}^{2}}{\mathrm{~m}_{-} \omega^{2}}+\frac{\mathrm{n}_{-}}{\gamma^{3} \mathrm{~m}_{-}\left(\omega / \mathrm{k}-\overline{\mathrm{v}}_{-}\right)^{2}}=\frac{\lambda^{2}+\dot{k}^{2}}{\left[1-(\omega / \mathrm{kc})^{2}\right] 4 \pi \mathrm{e}^{2}} \text {. }
$$

This equation may be transformed, with the following definitions,

$\nu_{-}=n_{-} \frac{\pi a^{2} e^{2}}{m_{-} c^{2}}, \nu_{+}=n_{+} \frac{\pi a^{2} e^{2}}{m_{-} c^{2}}, \frac{\omega}{k c}=\beta_{\phi^{\prime}}, \frac{\bar{v}}{c}=\beta_{0}, m_{+}=M, m=m, \lambda a=\psi$,

giving

$$
\frac{4 \nu}{\gamma^{3}} \frac{1}{\left(\beta-\beta_{0}\right)^{2}}+4 \nu+\frac{m}{M} \frac{1}{\beta_{\phi}^{2}}=\frac{\psi^{2}+\left(1-\beta_{\phi}^{2}\right) k^{2} a^{2}}{1-\beta_{\phi}^{2}}
$$

This is just Budker's $\mathrm{s}^{5}$ Eq. (27). The quantity $\psi$ is obtained from Eq. (161), which in this notation becomes

$$
\frac{\psi \mathrm{J}_{\mathrm{m}+1}(\psi)}{\mathrm{J}_{\mathrm{m}}(\psi)}=\frac{\mathrm{xK}_{\mathrm{m}+1}(\mathrm{x})}{\mathrm{K}_{\mathrm{m}}(\mathrm{x})}, \quad \mathrm{x} \equiv \kappa \mathrm{a}=\mathrm{ka}\left(1-\beta_{\phi}^{2}\right)^{1 / 2}
$$


Thus $\psi=\psi(x)$. Setting $\mathrm{m}=0$ in $\mathrm{Eq}$. (197) gives Budker' $\mathrm{s}^{5}$ second equation. His equation contains a minus sign on the R. H. S. of Eq. (197) which is an error.

Budker obtains the form of Eq. (196) for the two limiting cases $x>1$ and $x<1$. Consider first $x>1$. Then the R. H. S. of Eq. (197) approaches $x$, and the solution of Eq. (197) must be near the zeros of $\mathrm{J}_{\mathrm{m}}(\psi)$. Hence for small $\mathrm{m}$ and for the small zeros, $\psi \ll \mathrm{x}$ and may be neglected in the R. H. S. of Eq. (196), which then becomes

$$
\frac{4 \nu}{\gamma^{3}} \frac{1}{\left(\beta_{\phi}-\beta_{0}\right)^{2}}+4 \nu+\frac{m}{\mathrm{M}} \frac{1}{\beta_{\phi}^{2}}=\mathrm{k}^{2} \mathrm{a}^{2} .
$$

This is the usual "two-stream" equation.

Consider next the limit of the above equations when $x \ll 1$. Keeping the first few terms of the Bessel functions $K_{0}(x)$ and $K_{1}(x)$ from McLachlan, 28 p. 203, we obtain

$$
\begin{aligned}
& \mathrm{K}_{0}(\mathrm{x}) \approx-\gamma-\log \frac{\mathrm{x}}{2} \equiv \mathrm{L} \quad(\gamma=0.577) \\
& \mathrm{K}_{1}(\mathrm{x}) \approx \frac{1}{\mathrm{x}}+\frac{\mathrm{x}}{2}\left(\gamma-\frac{1}{2}+\log \frac{\mathrm{x}}{2}\right)=\frac{1}{\mathrm{x}}-\frac{\mathrm{x}}{2}\left(\mathrm{~L}+\frac{1}{2}\right)
\end{aligned}
$$

Setting $\mathrm{m}=0$ in Eq. (197) and using these equations we.obtain

$$
\frac{\psi \mathrm{J}_{1}(\psi)}{\mathrm{J}_{0}(\psi)}=\frac{\mathrm{xK}_{1}(\mathrm{x})}{\mathrm{K}_{0}(\mathrm{x})}=\frac{1-\frac{\mathrm{x}^{2}}{2}\left(\mathrm{~L}+\frac{1}{2}\right)}{\mathrm{L}} .
$$

Since $L \approx \log 1 / \mathrm{x}$, we may assume $\mathrm{x}$ so small that

$$
\mathrm{x}^{2} \mathrm{~L}<1, \quad \mathrm{~L} \geq 1
$$


We may obtain a consistent solution of (200) by assuming that $\psi$ is small, so that $J_{0}(\psi) \approx 1$ and $J_{1}(\psi) \approx \psi / 2$. Then Eq. (200) may be written as

$$
\psi^{2} / 2=1 / L \text {. }
$$

With this result, since $x^{2} \equiv\left(1-\beta_{\phi}^{2}\right) k^{2} a^{2}$, and using Eq. (201), we may neglect $x^{2}$ in the R.H.S. of Eq. (196), which now becomes

$$
\frac{2 \nu-}{\gamma^{3}\left(\beta_{\phi}-\beta_{0}\right)^{2}}+2 \nu+\frac{m}{\mathrm{M}} \frac{1}{\beta_{\phi}^{2}}=\frac{1}{L\left(1-\beta_{\phi}^{2}\right)}
$$

From Eq. (199) we may write L explicitly as

$$
L^{\prime}=\log \frac{1}{\mathrm{ka}\left(1-\beta_{\phi}^{2}\right)^{1 / 2}}+\log 2-\gamma .
$$

If $x>1$, it may be possible to neglect the terms $\log 2-\gamma$. In any case Budker's definition $L=\log k a\left(1-\beta_{\phi}^{2}\right)^{1 / 2}$ is incorrect, with the omission of a minus sign again. Otherwise Eq. (203) is identical with Budker's ${ }^{5}$ Eq. (32).

Budker's results are less general than ours because he considers only $\mathrm{m}=0$, and since no branch cut terms appear in his results, they are strictly valid only for growing waves. Needless to say, his derivation is not given in his paper.

Next we turn to the results of Dawson and Oberman. ${ }^{3}$ They consider a stationary plasma at zero temperature and look for normal modes, i. e., real frequencies that exist a long time after the initial perturbation. Therefore they do not obtain the branch cut term of Eq. (152) which decays with time.'

Their dispersion relations may be obtained from Eqs. (194) and (197). Since the plasma is stationary, $\bar{v}=0, \gamma=1$, and also $n_{+}=n_{-}$in Eq. (194). The first term on the R. H.S. may be neglected as $m_{+} \gg m_{-}$, so that the equation may be written as

$$
\lambda^{2}=\left(\omega_{p}^{2} / \omega^{2}-1\right)\left(k^{2}-\omega^{2} / c^{2}\right) \text {. }
$$


We have used the definitions for $\omega_{p}^{2}$ in Eq. (181) and $\kappa^{2}$ in Eq. (57). With the notation changes $\lambda \rightarrow \lambda^{i}, \omega_{p} \rightarrow \Omega, k \rightarrow \nu$, this becomes Eqs. (69) and (70) of Dawson and Oberman. ${ }^{3}$

The other dispersion relation given by Dawson and Oberman, ${ }^{3}$ their Eq. (80), for the determination of $\lambda$ is

$$
\lambda^{i} R \frac{J_{m}^{\prime}\left(\lambda^{i} R\right)}{J_{m}\left(\lambda^{i} R\right)}=\left|\lambda^{0}\right| R \frac{K_{m}^{\prime}\left(\left|\lambda^{0}\right| R\right)}{K_{m}\left(\left|\lambda^{0}\right| R\right)}
$$

We will show that this equation may be obtained from Eq. (161), showing that their results are a special case of our dispersion relations. Equation (161) is identical with Eq. (75), which was shown to be identical with Eq. (74), which is

$$
\lambda a \frac{J_{m}^{\prime}(\lambda a)}{J_{m}(\lambda a)}=\kappa a \frac{K_{m}^{\prime}(\kappa a)}{K_{m}(\kappa a)}
$$

Dawson and Oberman point out that their relation applies when $\lambda_{0}{ }^{2} \equiv \omega^{2} / c^{2}-\nu^{2}$ is negative. Hence $\left|\lambda_{0}\right|=\sqrt{-\lambda_{0}^{2}}=\left(\nu^{2}-\omega^{2} / c^{2}\right)^{1 / 2}$ or in our notation, $\left|\lambda_{0}\right|=\left(\mathrm{k}^{2}-\omega^{2} / \mathrm{c}^{2}\right)^{1 / 2} \equiv \kappa$. With these changes of notation, i. e., $\mathrm{R} \rightarrow \mathrm{a}$, $\lambda^{i} \rightarrow \lambda$, and $\left|\lambda_{0}\right| \rightarrow \kappa$, Eq. (206) is identical with Eq. (207). We have thus obtained the results of Dawson and Oberman from our dispersion relations.

Dawson and Oberman consider also steady-state situations which correspond to incoming waves from $r=\infty$, plus outgoing waves from the 'plasma. (For large $r$, these waves behave like $\left.e^{i \omega t}\left(e^{i \lambda_{0}^{r}} \pm e^{-i \lambda} 0^{r}\right) \frac{1}{\sqrt{r}}\right)$. These are their cases $A$ and $C$. There are no dispersion relations for these cases because the plasma is not the sole source of the waves. Our initial value treatment is evidently more suitable for treating waves due to the plasma. 
We consider next Sturrock's ${ }^{6}$ result for the dispersion relation of $a$ thin beam passing through a plasma. Let us treat the plasma as a dielectric, as Sturrock does. We must now go back to the original equations for the potentials, Eqs. (16) and (17), and replace e, the electric charge, by e/ $\epsilon$, where $\epsilon$ is the dielectric constant ${ }^{35}$ of the plasma:

$$
\epsilon=1-\omega_{p}^{2} / \omega^{2}
$$

The dispersion relation for thin beams, i. e., $k a\left(1-\frac{\omega}{\mathrm{kc}}^{2}\right)^{1 / 2} \ll 1$, has been derived above and is Eq. (203). Since the beam consists of electrons only, $\nu_{+}=0$, and replacing e by $\mathrm{e} / \epsilon$ in the expression for $\nu_{-}$, and rewriting the equation with the definitions of Eqs. (195) and (181), we obtain

$$
\frac{\omega_{-}^{2} a^{2}}{\epsilon\left(\omega / k-\bar{v}^{2}\right)}=\frac{2}{L\left[1-(\omega / k c)^{2}\right]}
$$

With the given definition for $\epsilon$, this equation may be put in the form

$$
\frac{\omega_{p}^{2}}{\omega^{2}}+\frac{\omega_{-}^{2}\left[1-\left(\frac{\omega}{\mathrm{kc}}\right)^{2}\right] \mathrm{k}^{2} \mathrm{a}^{2} \frac{\mathrm{L}}{2}}{(\omega-\mathrm{k} \overline{\mathrm{v}})^{2}}=1
$$

In the limit where $\omega / k c \ll 1$, this is Sturrock's Eq. (A.12). His expression for $\mathrm{L}$ is given by Eq. (204), with $\omega / \mathrm{kc} \equiv \dot{\beta}_{\phi} \ll 1$, and without the form $\log 2$. The neglect of $\log 2$ is presumably due to his crude approximation of an integral. We have thus derived Sturrock's results and shown that they are valid only when $\omega / \mathrm{kc} \ll 1$. Equation $(210)$ is, however, valid for arbitrary $\omega / \mathrm{kc}$, as long as

$$
\mathrm{ka}\left[1-\left(\frac{\omega}{\mathrm{kc}}\right)^{2}\right]^{1 / 2} \ll 1
$$


Consider next the two-stream equation for cold relativistic streams obtained by Finkelstein and Sturrock. ${ }^{14}$ For thin beams, their equation may be written (with minor changes of notation) as

$$
\frac{2 \nu_{-}}{\gamma^{3}\left(\omega / \mathrm{kc}-v_{-} / c\right)^{2}}+\frac{\mathrm{m}}{\mathrm{M}} \frac{2 \nu_{+}}{\left(\omega / \mathrm{kc}-v_{+} / c\right)^{2}}=\frac{1}{\ln 1 / \mathrm{ak}} .
$$

This equation agrees with Eq. (203), aside from the $v_{+} \neq 0$ term, only when $\omega / \mathrm{kc}<1$. The log expression is then almost the same as Eq. (204), but Finkelstein and Sturrock have neglected the two terms $\log 2-\gamma$. We have thus derived Eq. (211) and shown that it too is valid only for $\omega / \mathrm{kc} \ll 1$.

Additional work on the cold plasma has been done by Trivelpiece and Gould, ${ }^{31}$ who consider a cold cylindrical plasma and obtain the real frequency solutions when the plasma is surrounded by a conducting cylinder. Their dispersion relation (Eq. (17) in their paper) is identical with Eq. (205) above, with some changes in notation. Since the boundary conditions are different, the equation corresponding to Eq. $(207)$ is $J_{m}(\lambda a)=0$. They examine the solutions for this case in detail. Since only real frequencies are considered, $\omega / k c<1$. They consider also the situation where there is a dielectric cylinder between the plasma and the conductor, and obtain dispersion relations when $\omega / \mathrm{kc} \ll 1$ for arbitrary magnetic field. (We have assumed infinite $B_{0}$.) Trivelpiece and Gould ${ }^{31}$ list further references on the cold stream problem. Granatstein, Schlesinger, and Vigants ${ }^{32}$ consider a cold plasma and real frequency solutions $(\omega / \mathrm{kc}<1$, but not negligible). Their plasma cylinder is free, as in our case, and they obtain the results of Dawson and Oberman ${ }^{3}$ for infinite axial $B_{0}$. Some explicit dispersion curves are presented for this case. They also obtain the dispersion relation for zero axial field with the boundary condition of no surface motion. 


\section{DISCUSSION}

We have obtained the dispersion relation for relativistic streams with finite radius, under the assumption of pure longitudinal motion. We have also shown how the work of other authors, who make more simplifying assumptions, may be derived from our equation. For application to possible radiation from growing oscillations fed by an instability, we have also calculated the potentials $\phi$ and $A_{z}$ outside the plasma. Further work is in progress to obtain the solutions of Eqs. (159)-(161) for two-stream and simple velocity distributions.

We shall close this paper with a brief physical description of the reason for the occurrence of the distinctively new terms in our dispersion relation.

The new term which appears in the dispersion relation, Eq. (159), is the factor $\left[1-(\omega / k c)^{2}\right]\left[1 /\left(\lambda^{2}+\kappa^{2}\right)\right]$. Comparison of the R.H.S. of Eqs. (196), (198), and (203) shows that this factor has the two limits

$$
\begin{aligned}
& {\left[1-\left(\frac{\omega}{\mathrm{kc}}\right)^{2}\right] \frac{1}{\lambda^{2}+\kappa^{2}} \stackrel{ }{\kappa a>1} \frac{1}{\mathrm{k}^{2}}} \\
& \overrightarrow{k a \ll 1}\left[1-\left(\frac{\omega}{k c}\right)^{2}\right] \frac{L_{a}^{2}}{2} .
\end{aligned}
$$

We will show that these two limits correspond to two expressions for the electric field in terms of the charge distribution, one for a thick beam and the other for a thin beam.

In the usual derivations ${ }^{1,2}$ of the dispersion relation, one obtains the value of the electric field from the equation

$$
\nabla \cdot \mathrm{E}=4 \pi \rho .
$$

When the field is longitudinal, and $E=E_{k} e^{i k z}$, this equation gives

$$
E=\frac{4 \pi \rho}{i k} \text {. }
$$


This simple form of the equation leads to the dispersion relation, Eq. (159), with the simple form of Eq. (212) for the factor. Let us derive Eq. (215) from a different point of view. Equation (214) for the determination of the electric field takes on a particularly simple form in the static case. When the beam is thick and the charge sheets are close together these sheets are almost like infinite plane sheets for which one has, in the static case, $E=2 \pi \sigma$ ( $\sigma$ is the charge per unit area). Consider the case where all quantities vary as $e^{i k z-i \omega t}$, $k$ and $\omega$ real. A coordinate system moving with velocity $v=\omega / k$ will see all quantities stationary. Although the particles move, the charge distribution in this frame is stationary. In this system, we may apply the equation $E^{\prime}=2 \pi \sigma^{\prime}$. (The prime refers to the beam system.) The longitudinal electric field is invariant under Lorentz transformations. Hence in the lab system $E=E^{\prime}=$ $=2 \pi \sigma^{\prime}$. Since $\sigma^{\prime}$ is also invariant, $E=2 \pi \sigma$. Approximately, $\sigma \approx \rho / k$, so that

$$
\mathrm{E} \approx 2 \pi \frac{\rho}{\mathrm{k}}
$$

This is seen to be approximately the same result as Eq. (215). Although in this case of a thick beam, the electric field equation has the same form in all moving frames, we will see that in the next case, a thin beam, this is not true.

Consider now the thin beam problem. In order to find the electric field, we again go to a moving frame in which all charges appear stationary. We use Eq. (214). Since the beam is thin, the charges appear as charge lumps, and the equation gives

$$
\mathrm{E}^{\prime} \approx \frac{\mathrm{Q}^{\prime}}{\mathrm{d}^{\prime 2}} \approx \mathrm{Q}^{\prime} \mathrm{k}^{\prime 2} \approx\left(\frac{\pi \mathrm{a}^{2} \rho^{\prime}}{\mathrm{k}^{\prime}}\right) \mathrm{k}^{\prime 2}
$$


Again, since the longitudinal fields are invariant, $E=E^{\prime}$. The quantity $\sigma=\rho^{\prime} / k^{\prime}=\rho / k$ is also invariant, henee $E=\left(\pi a^{2} \rho / k\right) k^{\prime 2}$. To obtain $k$ in the lab system we note that $\mathrm{k}=2 \pi / \lambda$ and that $\lambda$ transforms like length. Since lengths in the moving system appear shorter in the lab system, we have

$$
\mathrm{k}^{\prime 2}=\mathrm{k}^{2}\left[1-\left(\frac{\omega}{\mathrm{kc}}\right)^{2}\right] \text {. }
$$

Thus we obtain for the electric field in the lab system, in terms of lab quantities,

$$
\mathrm{E} \approx\left(\pi \mathrm{a}^{2} \frac{\rho}{\mathrm{k}}\right) \mathrm{k}^{2}\left[1-\left(\frac{\omega}{\mathrm{kc}}\right)^{2}\right] .
$$

Collecting the two results Eqs. (216) and (219), we have

$$
\begin{aligned}
& E \underset{\kappa a \gg 1}{k} 2 \pi \rho \mathrm{k}\left(\frac{1}{\mathrm{k}^{2}}\right) \text {, } \\
& \left.E \underset{\kappa a \ll I}{2} 2 \pi \rho \mathrm{k}\left\{\frac{\mathrm{a}^{2}}{2}\left[1-\frac{\omega}{\mathrm{kc}}\right)^{2}\right]\right\} \text {. }
\end{aligned}
$$

Since Eq. (215) or its similar form Eq. (220) leads to the R. H.S. of Eq. (212), one sees that Eq. (220) would give approximately the R.H.S. of Eq. (213) for the factor in the dispersion relation, Eq. (159). Thus we have shown, although crudely, that the forms (212) and (213) are due to the different relations of the electric field to the charge distribution for thick and thin beams.

The occurrence of the inequality $\kappa a \gg 1$ or $\kappa a<<1$ is now understood. By Eq. (218) we see that $\kappa \equiv k^{\prime}$ is the wave number in the moving or beam frame. Hence it is the wave number in the beam frame that determines the appropriate limit of the dispersion relation. 
$-72-$

Acknowledgments

I should like to thank Prof. G. Schmidt for many valuable comments, criticisms, and guidance. I am also indebted to Dis. A. Kaufman and W. Newcomb for many illuminating and enlightening conversations, to Prof. D. Finkelstein for his valuable remarks, and to Dr. W. Heckrotte for helpful encouragement. I should like to thank the Lawrence Radiation Laboratory, Livermore, California, for its kind hospitality while this work was performed. 


\section{REFERENCES}

"Work done under the aitgpices of the U. S. Atomia Energy Commisaion.

${ }^{1}$ L. D. Landau, J. Phys. USSR 10, 25 (1946).

2 J. D. Jackson, J. Nucl. Energy, Part C 1, 171 (1960).

3 J. Dawson and C. Oberman, Phys. Fluids $\underline{2}, 103$ (1959).

${ }^{4}$ S. A. Bludman, K. M. Watson, and M. N. Rosenbluth, Phys. Fluids 3 ; 747 (1960).

${ }^{5}$ G. I. Budker, At. Energ. USSR 1 , No. 5, 9 (1956). [Translation abridged with many errors - CERN Symposium 1, 68 (1956)].

${ }^{6}$ P. A. Sturrock, Phys. Rèv. 117,1426 (1960).

${ }^{7}$ T. H. Stix, Theory of Plasma Waves (McGraw-Hill, N. Y., 1962), pp. $154-155,223$ !

${ }^{8}$ D. A. Tidman, Phys. Revi. 117; 366 (1960); I. B. Bernstein and S. K. Trehan, Nucl. Fusion 1, 3 (1960); G. B. Field, Astrophys. J. 124, 555 (1956).

${ }^{9}$ G. Schmidt and G. Halpern, Phys. Rev. 131, 217 (1963); G. Schmidt, Phys. Fluidsi $\underline{7}, 915$ (1964).

${ }^{10}$ L. Brillouin, Wave Propagation (Academic Press, N. Y., 1960).

11 J. A. Stratton, Electromagnetic Theory (McGraw-Hill, N. Y., 1941), pp. $333-340$.

${ }^{12}$ R. W. Landau and G. Schmidt, Stevens Institute of Technology, Hoboken, N. J., Mar.! 1964, SIT-119 (ưnpublished). 
13 O. Buneman, in Plasma Physics, ed. by J. E. Drummond (McGraw-Hill, N. Y, 1061), ehap, 10, pp, 272, 287.

${ }^{14}$ D. Finkelstein and P. Sturrock, in Plasma Physics, ibid., pp. 226-228.

${ }^{15}$ A covariant derivation has been given by S. T. Belyeav and G. I. Budker, Doklady Akad. Nauk SSSR 107, 807 (1956); Soviet Phys. "Doklady" English Transl. 1, 218 (1957).

${ }^{16}$ G. N. Watson, Theory of Bessel Functions (University Press, Cambridge, 1948), 2nd ed.

${ }^{17}$ An excellent review of transform theory is given by I. A. Sneddon, in Handbuch der Physik, ed. by S. Flugge (Springer-Verlag, Berlin, 1955), vol. 2, pp. 198-347.

${ }^{18}$ G. Backus, J. Math. Phys.. $1,178(1960)$.

19 Reference 16, pp. 596-602.

${ }^{20}$ R. Courant and D. Hilbert, Methods of Mathematical Physics (Interscience, N. Y., 1953) vol. 1, pp. 70-71.

21 Reference 16, p. 602.

22 E. C. Titchmarsh, Theory of Fourier Integrals (Clarendon Press, Oxford, 1948), 2nd Ed., pp. 42, 14.

${ }^{23}$ G. A. Campbell and R. M. Foster, Fourier Integrals for Practical Applications' (Van Nostrand, New York, 1948).

${ }^{24}$ We are indebted to G. Halpern for indicating that Eq. (50) may be integrated in a closed form. 
25 A. Erdelyi, Tables of Integral Transforms (McGraw-Hill, N. Y., 1954) vol. 1, No. 27 , p. 17 .

${ }^{26}$ A. Erdelyi, Higher Transcendental Functions (McGraw-Hill, N. Y. , 1953), vol. 2, Eq. (35), p. 102.

${ }^{27}$ J. A. Stratton, Electromágnetic Theory (McGraw-Hill, N. Y., 1941), 1st Ed., pp. $372-374$.

${ }^{28}$ N. W. McLachlan, Bessel Functions (Clarendon Press, Oxford, 1955), 2nd Ed., pp. 190-214.

29 Reference 28, p. 203.

30 Reference 16, p. 511.

${ }^{31}$ A. W. Trivelpiece and R.: W. Gould, J. Appl. Phys. 30, 1784 (1959).

32 V. L. Granatstein, S. P." Schlesinger, and A. Vigants, IEEE Trans. $\underline{A P-11}, 489$ (1963).

33 Reference 4, Eq. (2.19), p. 750.

${ }^{34}$ Reference 4, Eq. (2.2a).

${ }^{35}$ See, e.g. , ref. 7, p. 10.

/ no 


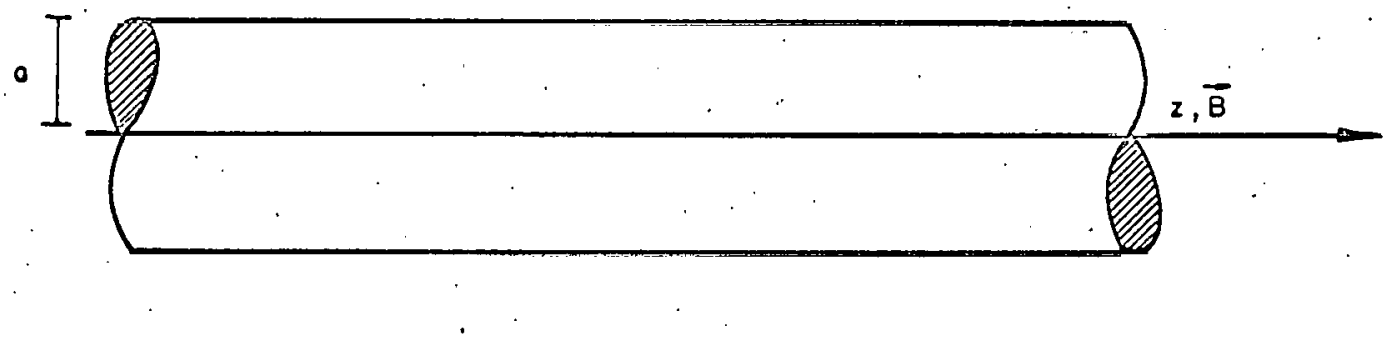

Fig. 1. Cylinder geometry. 


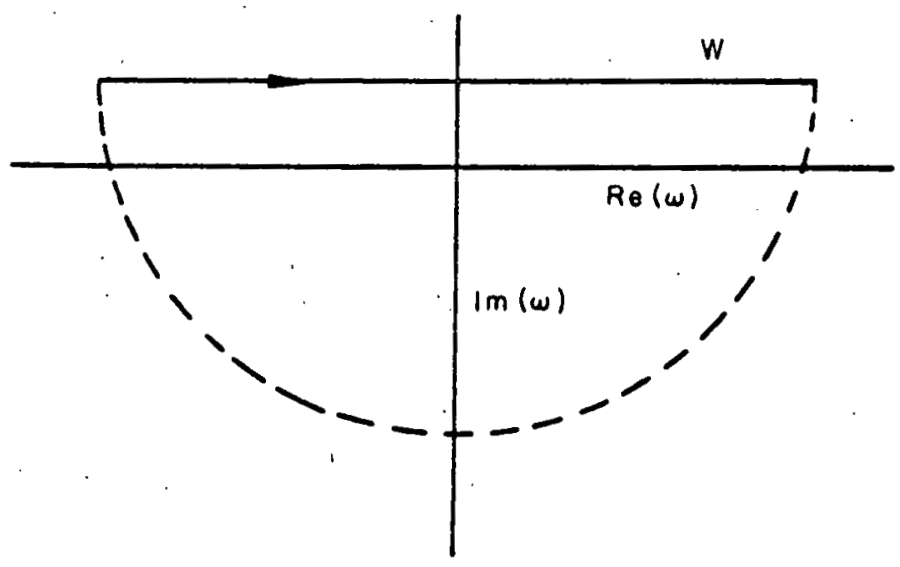

Fig. 2. Contour for Laplace transform part of Eq. (36). 

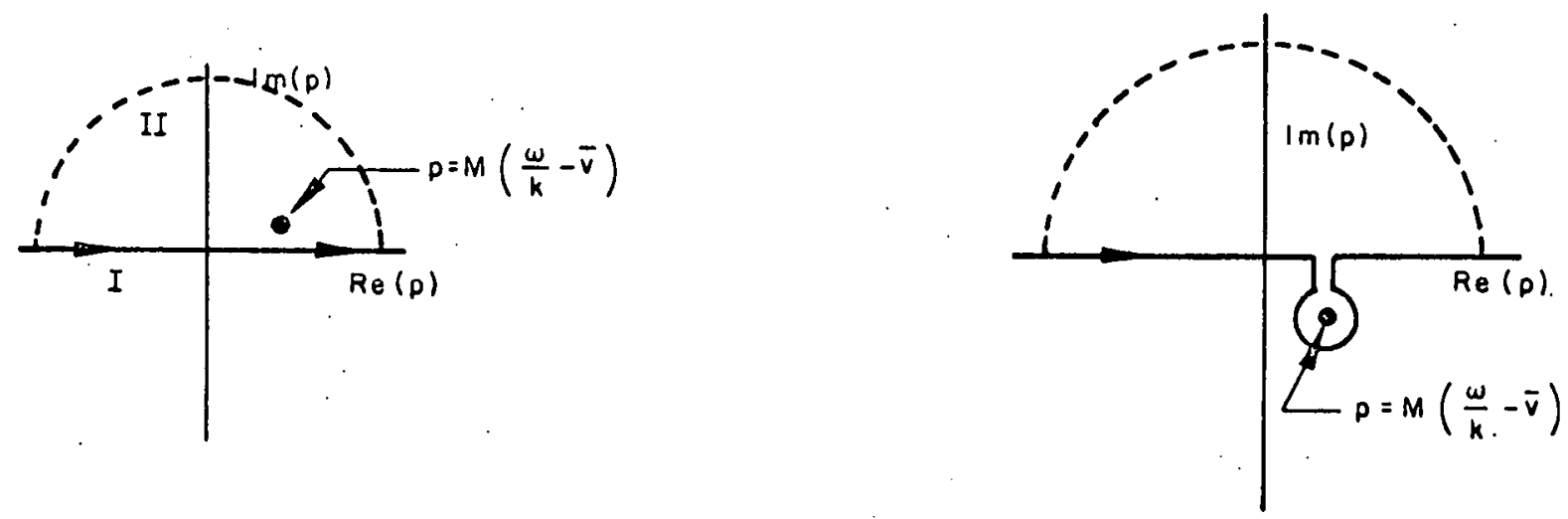

Fig. 3. Integration contour in the complex p-plane. $\operatorname{Im}(\omega)>0, k>0$.
Fig. 4. Integration contour in the complex $\mathrm{p}$-plane. $\operatorname{Im}(\omega)<0, \mathrm{k}>0$. 


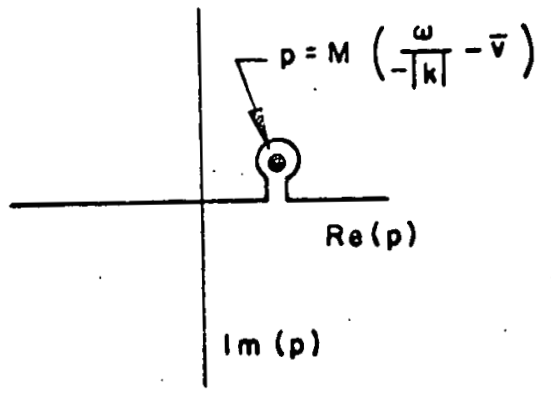

Fig. 5. Integration contour in the complex $\mathrm{p}$-plane. $\operatorname{Im}(\omega)<0, \mathrm{k}<0$. 


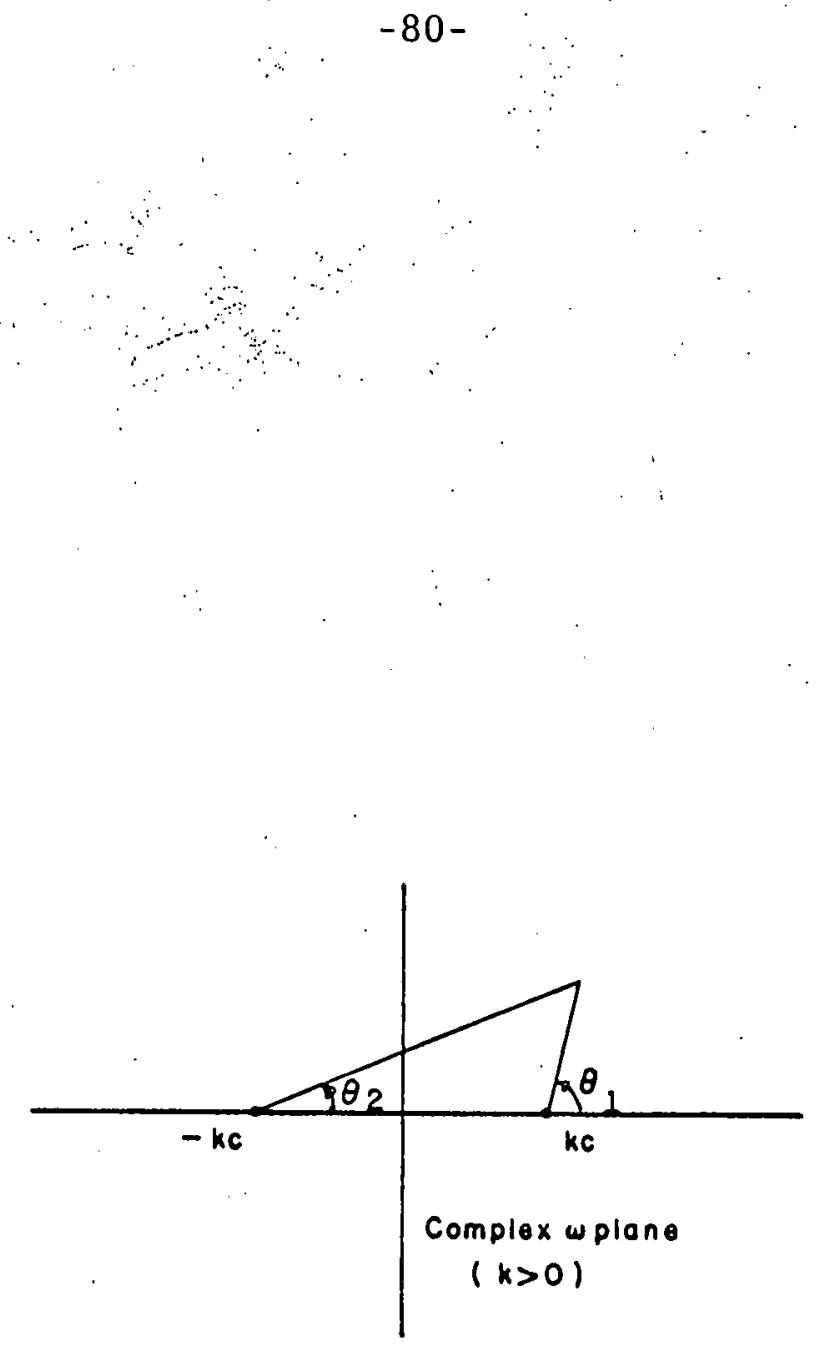

Fig. 6. Diagram illustrating range of $\theta_{\mathrm{L}}$. 


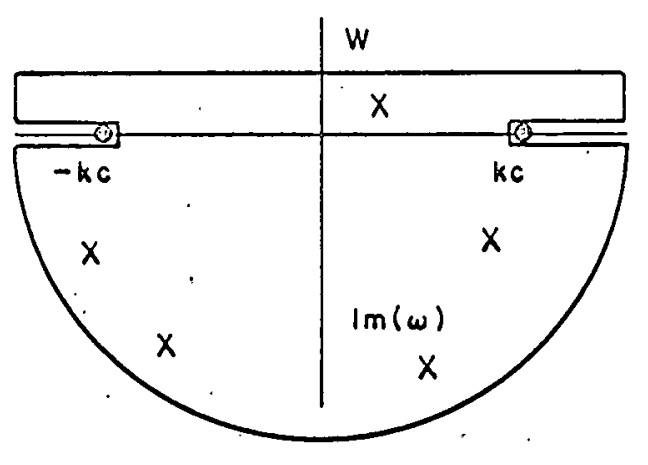

Fig. 7: Integration contour in the complex $\omega$-plane, $\mathrm{k}>0$. 
This report was prepared as an account of Government sponsored work. Neither the United States, nor the Commission, nor any person acting on behalf of the Commission:

A. Makes any warranty or representation, expressed or implied, with respect to the accuracy, completeness, or usefulness of the information contained in this report, or that the use of any information, apparatus, method, or process disclosed in this report may not infringe privately owned rights; or

B. Assumes any liabilities with respect to the use of, or for damages resulting from the use of any information, apparatus, method or process disclosed in this report.

As used in the above, "person acting on behalf of the Commission" includes any employee or contractor of the commission, or employee of such contractor, to the extent that such employee or contractor of the Commission, or employee of such contractor prepares, disseminates, or provides access to, any information pursuant to his employment or contract with the Commission, or his employment with such coritractor. 